\title{
Two deductions: (1) from the totality to quantum information conservation; (2) from the latter to dark matter and dark energy
}

\author{
Vasil Penchev, vasildinev@gmail.com \\ Bulgarian Academy of Sciences: Institute of Philosophy and Sociology: \\ Dept. of Logic and Philosophy of Science
}

\begin{abstract}
The paper discusses the origin of dark matter and dark energy from the concepts of time and the totality in the final analysis. Though both, and especially the latter, seem to be rather philosophical, nonetheless they are postulated axiomatically and interpreted physically, and the corresponding philosophical transcendentalism serves heuristically. The exposition of the article means to outline the "forest for the trees", however, in an absolutely rigorous mathematical way, which to be explicated in detail in a future paper. The "two deductions" are two successive stage of a single conclusion mentioned above. The concept of "transcendental invariance" meaning ontologically and physically interpreting the mathematical equivalence of the axiom of choice and the well-ordering "theorem" is utilized again. Then, time arrow is a corollary from that transcendental invariance, and in turn, it implies quantum information conservation as the Noether correlate of the linear "increase of time" after time arrow. Quantum information conservation implies a few fundamental corollaries such as the "conservation of energy conservation" in quantum mechanics from reasons quite different from those in classical mechanics and physics as well as the "absence of hidden variables" (versus Einstein's conjecture) in it. However, the paper is concentrated only into the inference of another corollary from quantum information conservation, namely, dark matter and dark energy being due to entanglement, and thus and in the final analysis, to the conservation of quantum information, however observed experimentally only on the "cognitive screen" of "Mach's principle" in Einstein's general relativity therefore excluding any other source of gravitational field than mass and gravity. Then, if quantum information by itself would generate a certain nonzero gravitational field, it will be depicted on the same screen as certain masses and energies distributed in space-time, and most presumably, observable as those dark energy and dark matter predominating in the universe as about $96 \%$ of its energy and matter quite unexpectedly for physics and the scientific worldview nowadays. Besides on the cognitive screen of general relativity, entanglement is available necessarily on still one "cognitive screen" (namely, that of quantum mechanics), being furthermore "flat". Most probably, that projection is confinement, a mysterious and ad hoc added interaction along with the fundamental tree ones of the Standard model being even inconsistent to them conceptually, as far as it need differ the local space from the global space being definable only as a relation between them (similar to entanglement). So, entanglement is able to link the gravity of general relativity to the confinement of the Standard model as its projections of the "cognitive screens" of those two fundamental physical theories.
\end{abstract}

Key words: confinement, dark energy, dark matter, entanglement, general relativity, physical and mathematical transcendentalism, quantum information, the Standard model, transcendental invariance 
I THE PHILOSOPHICAL AND CULTURAL CONTEXT OF THE COMPOSITE DEDUCTION

The main philosophical and cultural sense of inferring new physical phenomena such as dark matter (Trimble 1987: 451-452) and dark energy (Riess et al. 1998: 1009) by postulating totality physically, and formally and mathematically consists in restoring the speculative approach to experimental science (such as physics) as well as a continuous and smooth "bridge" between the following two poles cut by Descartes's dualism: the pole of the sensual, empirical, experimental, and physical (i.e. the "body" or the "object" of classical German philosophy) versus the pole of mental, speculative, postulable, and mathematical (i.e. the "mind" or the "subject").

Thus, the composite deduction being properly a deduction infers a statement belonging to the realm of physics from a speculative postulate belonging to metaphysics, but formulated only mathematically, in a rigorous, formal and logical way.

An echo and reverberation of Descartes's dualism predetermined fundamentally the direction of Western science and philosophy for developing is the neglect and oblivion of metaphysics (e.g. reflected in Heidegger's “oblivion of the being") as a fundamental element of any objective cognition including scientific.

Cognition, science, and particularly physics have been related to philosophy and metaphysics since the rise of philosophy, mathematics, and science in Ancient Greece. The relation and proportion of the empirical and speculative has been changed slowly during centuries and millennia culminating to the almost absolute predomination of the former nowadays. The "motion of the pendulum" has reached the one extreme, that of the absolute domination of the one Descartes's "pole", the "body". In the framework of his dualistic metaparadigm the transition is possible only by history including scientific and only as a pendulum-like combination of those two inconsistent polarities.

If one keeps the "pendulum-like metaparadigm" implied by dualism, a historical movement in opposite direction would be to be expected, i.e. to speculative metaphysics, including in science. If one adopts Hegel's revision of the same paradigm consisting of in the historical, ontological, and even logical "synthesis", the unification of the metaphysical (particularly, as the "totality") and physical (in particular, as "dark matter" and "dark energy") might be admitted.

The present paper traces that unification in virtue of a formal and mathematical deduction.

\section{KANT'S “COPERNICAN REVOLUTION” OF TRANSCENDENTALISM}

Kant devoted his works to heralding an absolutely new viewpoint to philosophy estimated by himself coining the metaphor of "Copernicus revolution" and designated by the then neologism of "transcendentalism" also coined by him meaning a new reading of transcendence and the transcendent in virtue of what the "totality" should be meant philosophically, and the way, in which it should be defined. Indeed, his fundamental discovery appeared in the context of the then Western philosophy as well as a certain resolution of the problem of Descartes's dualism.

Nonetheless, his discovery is much more fundamental as far as it justifies the existence and constitution of philosophy by the concept of the totality specific just to it and its claim of absolute generality.

Meaning the development of philosophy, science and history since his age until nowadays, one can notice that the totality seemed to be an only metaphysical concept eventually postulable, but unverifiable practically whether empirically or experimentally.

Thus, it could be studied in philosophy or theology, and even in natural or experimental science, but in it, only by mediation of mathematics and its basic and constituting concept of infinity or the 
actual infinity of set theory, being able to reflect the philosophical idea of the totality, and possibly, the approach of transcendentalism.

Consequently, science had and has an access to metaphysics only by mediation of mathematics, and not otherwise, i.e. directly or by philosophy or theology. Even more: the eventual pathway of science to the totality via theology or speculative philosophy is absolutely prohibited as far as the opposition, and thus, gap between science and theology or speculative philosophe constitutes the fundamental option and possibility of cognition in our age, or its "episteme" in Foucault's sense.

Therefore, the opposition of "physics" (particularly, the science of "physics") and metaphysics (particularly, the doctrine of transcendentalism) is a dichotomy so basic in the contemporary culture that it does not allow for the doctrine of transcendentalism invented properly to overcome just that same abyss not to be able to be understood, on the one hand, and physics by itself generates "dark" entities, on the other hand, being unable to reflect its foundations, in the "blind spot, scotoma" of which they appear therefore remaining "dark" unavoidably and in definition.

So, the pathos of the present article is even rather enlightening since it calls for reflecting the foundations of the contemporary cognition, itself generating fundamental epistemological problems being paradoxically "visible as darkness" and thus preventing the further development of science.

III THE IDEA OF FORMAL, PHYSICAL AND MATHEMATICAL TRANSCENDENTALISM AND ITS UNITY WITH PHILOSOPHICAL TRANSCENDENTALISM

Once that is the real interrelation of physics and metaphysics in our age, a breakthrough of science to philosophy, and even maybe the only possible one, is by mediation of a formal and mathematical transcendentalism applied to physics, more exactly, to quantum mechanics as far as the access to the totality is admissible in the configuration of our episteme, first of all, trough infinity and through the actual infinity of set theory, i.e. by a relevant mathematical model in the final analysis, preferably rooted and justified in the foundations of mathematics explicitly.

One needs a formal and axiomatic definition of the totality, namely as "all" literarily, i.e. as what being all contains its externality within itself necessarily. Thus, the description of what is ostensibly out of the totality is also necessary doubled therefore doubling the description of anything as far what is ostensibly within the totality depicts an identical counterpart outside. So, the only effective result of introducing the totality formally as "all" is the doubling of everything and thus and particularly, the doubling implied as a fundamental problem after Descartes's dualism turns out to be easily explicable on the ground of Kant's identification of the transcendent (i.e. out of the totality) with its counterpart transcendental (i.e. within the totality). As to terms and concepts of the tradition of Western philosophy, one might mean that transcendental resolution exemplified to the "mind and body" or the "subject and object" of classical German philosophy, etc., but this is not necessary at all:

Instead of that philosophical discourse illuminated and even consecrated by tradition, one, quite blasphemous to it (but only at first glance), can mean an only formal and mathematical operation of doubling as an algebraic equivalent to the concept of the totality therefore adopting it as underlying and properly fundamental. The same idea of purely abstract and formally algebraic doubling may be found as embodied a long time ago in the concept and quantity of information as well as in its unit of an elementary choice, i.e. a "bit of information":

Indeed any bit of information is interpretable furthermore fundamentally and ontologically as a single and elementary "doubling" of the same kind. Then, the concept and even quantity of information would represent the idea of transcendentalism formally. If one adds the concept of 
infinity (respectively, "actual infinity") to information, which defines "quantum information" properly, a double referring to the totality would be available: by mediation of both "infinity" and "bit of information" turning out to be complete and consistent, i.e. a sense perfect and symmetric.

The same idea of formal transcendentalism needs still two specifications, parallel and thus independent of each other, to be applicable to the composite deduction mean here, namely:

1. The formal structure utilized in quantum mechanics: that of the separable complex Hilbert space interpreted as a qubit space.

2. The identification of the local and global space meant by the Standard model, but now interpreted much more fundamentally, in terms of the foundation of mathematics, those of set theory and arithmetic, as the equivalence of the well-ordering "theorem" and the axiom of choice: an equivalence provable in (ZFC) set theory.

The idea of formal transcendentalism can be explicated in each of them as follows:

1. The separable complex (qubit) Hilbert space in terms of formal transcendentalism:

Indeed, one can see it as pairs of dual qubits of the same name, and thus, anti-isometric to each other. Any qubit is interpreted as the generalization of "bit" referring to infinite series and sets, and any pair of dual anti-isometric qubits represents a usual, "classical" bit of information.

Thus, any pair of qubits embodies the formal model of the totality and implies formal and mathematical transcendentalism. In truth, any of the two twin qubits can represent the totality whether "inside" or "outside" if and only if its counterpart in the pair means oppositely, inversely, and idempotently again the totality correspondingly either "outside" or "inside" accordingly.

Any single qubit in the qubit Hilbert space can be interpreted as representing a state of infinity my means of a certain wave function (consisting only of that qubit and with zero coefficients of all others) as it is explained in detail in a previous paper (Penchev 2020 August 25). Thus, any single qubit as well as any series of qubits (thus necessary belonging to the qubit Hilbert space) are able to represent a state of the totality though seen mathematically as "infinity": i.e. a "state of infinity" as a certain wave function. The fact that as any single qubit as any series (whether infinite or finite) of qubits is able to represent any state of infinity (respectively, the totality) equally well seems to be paradoxical at first glance. It is due the extraordinary property of an infinite structure to be able to be isomorphic to a true substructure of it,

Summarizing, the transcendentality of the qubit Hilbert space can be found as "inside" as "outside" it. The former as a "state of infinity" (or a "state of the totality"), thus referring to the totality immediately, and the latter as the pair of two dual qubits of the same name and able to depict the pair of the totality itself and its counterpart ostensibly "external" to it but coinciding in the sense of antiisometry.

Any given physical space (e.g. what we dwell) is to be described by an additional relation or even ratio as what the fundamental Planck constant can be referred to. Its sense and meaning not less are how many quantum information would be to be equated to a unit, i.e. a bit of classical information. The same coefficient implies a few not less essential and practically important other interpretations:

The structure of Schrödinger equation (that is regardless of the values of the constants it) reflects the same relation of the "local and global space" (utilizing the terms developed in the Standard model and its philosophy rather) unambiguously. It shows how much "physical action" is equal to a unit of information whether quantum or classical. It elucidates even how much "infinity" (e.g. as a geometric unit) refers equivalently to an arithmetical unit (i.e. that notated standardly as "1"). The latter interpretation implies still one: how much of the "curved" should be equated to a unit of the "straight". 
All those interpretations are related to formal transcendentalism by the elucidated above relation of the qubit Hilbert space

2. The equivalence of the axiom of choice and the well-ordering theorem in terms of formal transcendentalism:

That equivalence is embedded in quantum mechanics as well, more exactly in the statement that its "vector hypostasis" and its "function hypostasis" are isomorphic to each other. In fact, they are actually isomorphic if and only if the equivalence meant in " 2 " is valid.

The last statement turned to be invested in the history in quantum mechanics, even its "birth" as the unification of matrix mechanics and wave ("undulatory") mechanics described in detail in another study (Penchev 2020 October 5) where the identifications of the local and global space in the Standard model, on the one hand, and in general relativity, on the other hand, are considered and furthermore identified in turn as a corollary from the same equivalence invested in Hilbert space and thus, in foundations of quantum mechanics.

Now, those identifications will be only summarized as a still one embedment of physical transcendentalism though implicit and not proclaimed as such usually:

The hypothetical transfer "trough" the transcendental boundary of the totality (which generates only a doubling effectively remaining within the totality once it has been defined as "all") implies as a necessary property for the "bandy" ("curved") to be "flattened". This is due to the implicit transition trough the "transcendental" boundary of infinity being identical to that of the totality after the mathematical consideration of transcendentalism, mathematical and physical properly already.

Indeed, passing "beyond" the totality, i.e. into the imaginary transcendent realm, one should reach the boundary of infinity meanwhile as the model of the pair of two dual qubit, each of which represents the totality after its doubling or respectively, either its externality or internality correspondingly. It seems to be obvious that reaching infinity is a necessary "contortion" (or condition) for the transcending into the other qubit.

Once infinity has been involved, it explains that "flattening" by the unique property of infinity to be isomorphic to true "parts" of itself: speaking in Dedekind's manner, infinity is what can coincide with its true part.

Then, the double opposition of the "flat" and "infinite" versus the "bandy" and finite can be reduced to a special kind of invariance callable "transcendental invariance" (as in the cited paper for its origin from physical and mathematical transcendentalism) under the condition of the equivalence mean in " 2 " (and thus, in quantum mechanics implicitly as it is elucidated both above and in the cited paper at issue).

Though seeming rather paradoxical at first glance, the following conclusion is valid: quantum mechanics implies that general relativity is the relevant theory of quantum gravity once physical and mathematical transcendentalism has been adopted as here.

The same equivalence " 2 " and the general property of idempotency being the definitive relation of any two dual entities of the same name (e.g. two dual qubits) implies the cyclicality of the totality, or particularly, that of the universe in physical terms. Physical transcendentalism as it can be found in the fundamental contemporary physical theories implies further a new relativity, that of the "big" and "small': loosely, the universe turns out to be within any quantum in turn within it therefore demonstrating that cyclical idempotency not only of the "big" and "small", but that of as "one" and "many" as "many" and "much" as well. That cyclical idempotency is a necessary condition for "quantum-information conservation" as it will be explained in the section ("VII") considering that new law of conservation. 


\section{TRANSCENDENTALITY AND MOTION, TRANSCENDENTALISM AND DIALECTICS, INFORMATION AND PHYSICAL ACTION}

Hegel was who introduced the concept of "philosophical motion" (known also as "ontological motion" or "logical motion") often reckoned to be a metaphor, even rather misleading. His idea, translated in terms of this paper, is that the totality being "all" generates a fundamental essence, a counterpart after any consideration claiming to be transcendental.

That philosophical essence means "change by itself" as if by some hidden internal cause rather than by any external cause. Indeed, any external cause should be excluded as to the totality being all. Nonetheless, the totality is not static, unchangeable, but on the contrary and by itself. This is due to, speaking loosely, the definitive necessity to integrate its "externality" within it. Hegel's papers demonstrate that this generates some change even on a purely logical level therefore contradicting the usual standard formal logic, more precisely, it contradicts the axiomatic property of "identity", and laws of "non-contradiction" and ""excluded middle".

In other words, Hegel's approach shows that any entity within the totality obeys classical logics, but the totality itself does not. Thus, he coined the idea of "dialectic logic" or "dialectics", being properly philosophical and therefore alternative to the classical one. Dialectics is natural logic, logic by itself, or "ontological logic". It is consistent though inconsistent to classical logic once Hegel's "dialectic laws" has been adopted. In contemporary terms, it is one of the first non-classical logics (if not the first one) and very famous, however rather among philosophers than among logicians.

Its main argument in the framework of the present context can be synthesized so. After the totality has generated fundamental pairs doubling anything: once coinciding with itself, but not less, twice differing from itself simultaneously. Thus, both statements are to be valid at the same time, but obviously contradicting the cited rules of classical logic. One can notice that both simultaneous dialectic statements are valid to any change, particularly to any mechanical motion within the totality. Then, to keep the last property to the totality itself and meaning the observed validity to it, one as Hegel can postulate that the totality moves by itself and thus herald "dialectics".

If "physical and mathematical transcendentalism" has been granted, one can suggest "physical and mathematical dialectics" meaning the following:

A generalized change (or mechanical motion, in particular) can be involved. After that is the case, the "transcendental invariance" of the present context can be linked to a dialectic counterpart, properly, "physical and mathematical dialectics" admissibly. In fact, the origin of Hegel's dialectics from Kant's transcendentalism can be repeated in the rigorous physical and mathematical terms and concepts so that their formal equivalents be demonstrated as two interpretations of the same underlying structure, once, doubling anything transcendentally, twice finding anything in dialectic (i.e. "philosophical") movement. The bridge being the formal, physical and mathematical counterpart of the philosophical one able to connect Kant and Hegel is simultaneously that link meant in the present paper to join quantum mechanics with general relativity, and thus, in the final analysis, the philosophical "first principle" of the totality, to "dark matter" and "dark energy" corroborated experimentally (The Nobel Committee 2011; Ade 2016) only in the end of the $20^{\text {th }}$ and the beginning of the $21^{\text {st }}$ century..

The same link, furthermore, can be demonstrated explicitly and expressively as follows:

The physical and mathematical transcendentalism was demonstrated already as embedded into the separable complex Hilbert space of quantum mechanics, and hence, passing into the identification of the local and global spaces of the Standard model. Thus, what follows is to be shown how the same mathematical structure can be considered as Hegel's (or even Marx's) "philosophical movement" 
keeping one thing in mind: the formal, physical and mathematical equivalent of that "philosophical movement" would be to be gravity (even just as it is reflected in general relativity) in the intention of the present paper.

For example, one can investigate any pair of dual qubits therefore complementary to each other in the sense and meaning of Niels Bohr. The dual qubits are anti-isometric to each other in the qubit Hilbert space and can be accepted as identical after the corresponding convention about anti-isometry. However, their simultaneous measurement is forbidden according the principle of complementarity ${ }^{1}$. After that is the case, the former statement implies that both qubits can be granted as the same (i.e. anti-isometric to each other), and the latter, that one forbids for the two qubits to be equated to each other in virtue of the complementarity (even on the same base of anti-isometry). So, this means that Hegel's "dialectic formula of movement" is valid to any pair of qubits, and thus, to the qubit Hilbert space in turn equivalent to the separable complex Hilbert space of quantum mechanics: it is simultaneously a formal structure embedding Hegel's "dialectic movement", which had to be proven.

Furthermore, the discussed pair of dual qubits possesses the structure of unit of information composed by the classical unit, a bit of information, and the quantum unit, a qubit of information. Quantum mechanics at all (or the Schrödinger equation particularly, but especially obviously) demonstrates that a certain variable quantity of physical action corresponds to any wave function being dimensionless physically for the Planck constant interpretable as how much of physical action is equal to a unit (presumably, a bit) of information.

Just according to Hegel's intention, his dialectics is able to prove that the material world (expressible by physical action) obeys the spiritual one (meant in the concept and quantity of information). However, contrary to his intention, the converse statement is not less true as far as the material and spiritual worlds are both equivalent and complementary to each other.

The unity of transcendentalism and dialects both eventually physical and mathematical has an unambiguous image in the unity of both most fundamental physical theories: quantum mechanics and general relativity. The latter continues to be elucidated in the present paper.

\section{THE POLE OF THE METAPHYSICAL TOTALITY FROM THE VIEWPOINT OF THE CONTEMPORARY PHYSICS AND MATHEMATICS}

The concept of the totality in philosophy is able to unify a few others in mathematics and physics, and especially in their foundations: enumerated from (1) to (11) further in the present section. Though, they seem to be too remote from each other, and on the top of that, belonging to different branches of science, their "family resemblance" becomes obvious once they have been related to the philosophical "totality" (involved rather implicitly only by the classical German philosophy). All of them seen from the viewpoint of the totality can help for the concept of "the physical and mathematical totality" (involved before that implicitly by the joint consideration of both mathematical and physical transcendentalism and mathematical and physical dialectics) to be outlined by the features most essential in the present context:

\footnotetext{
${ }^{1}$ The principle of complementarity is valid also even purely mathematically if one means the dual Hilbert space as the space of functionals (i.e. mapping a set of function into the set of complex numbers) where the set of functions (i.e. automorphisms of the set of complex numbers) is other Hilbert space, to which the former is dual. They cannot be available simultaneously in the logical meaning the the one is a necessary condition of the other. Nonetheless, this means that both dual Hilbert space are equivalent as far as each of them is a necessary condition of the other.
} 
(1) The universe as the physical totality: it can be depicted by the opposite trend in the contemporary physics and cosmology for the universe not be understood as the "totality of all" e.g. by means of many universes whether parallel or interacting with each other or the "multiverse" consisting of universes as its substructures whether isomorphic or only similar to it.

That trend can be understood very easily by the dominating requirement to physics, astronomy, and cosmology in our age to be empirical and experimental sciences. This a historical condition valid for those science in the Modern epoch, but not forever or definitively for them. In fact, all of them are unthinkable without relevant mathematical models, which by themselves are not empirically or experimentally verifiable and should be consistent only. Just for the "episteme" (in Foucault's sense) nowadays, the mathematical models being "secondary" obey outrightly the available empirical and experimental data, and on the top of that, Occam's razors cuts any additional property or relation, for which "no data".

Thus, the totality of the universe turns out to be rather "under a cloud" in the science of Modernity. Particularly, concepts rejecting the postulate of the total universe (i.e. the totality of the universe), such as those mentioned above, thrive and even overrun the rival of the total universe as far as our episteme helps them.

The totality and total universe are in disfavor keeping their links to metaphysics, demonstrated once again in this paper. Metaphysics just as theology or pseudoscience is opposed to science, ergo, the total universe as well particularly.

However, if an unverifiable statement implies empirically verifiable corollaries, and they be corroborated experimentally, this is an argument in favor of the tested unverifiable statement though it is postulated. This is usual practice in theoretical science nowadays. Following it, the present paper tries to reason the conjecture of the total universe by its corollaries eventually that both dark matter and dark energy follow from it exceptionally in the framework of the generally adopted postulates of quantum mechanics and general relativity. Indeed, new statements such as "quantum information conservation" or the "cyclic closeness of the universe" are articulated expressively, but they are only new theorems from the same axiomatic base, and thus, not involving any new postulates and not exchanging any of the available postulates by its negation or modification. Consequently, the conjecture of the total universe would be to be reckoned as "conservative" in the elucidated sense.

(2) The "Big Bang" as its "beginning" as the bound of the temporal totality:

Kant's transcendentalism and Hegel's dialectics reveal and preach that the properties and relations of the totality are fundamentally different from those of any parts, which, empirically and experimentally, are only accessible. The Big Bang is to be related to the totality (indeed, the Bing Bang itself or the "zero time of the universe" is inaccessible experimentally) and therefore investigable only by the tools of the mathematical and physical both transcendentalism and dialectics.

The Big Bang should be seen as a transcendental limit in the past so that "all before which" (being an "externality of the totality") be to be observed experimentally only in the empirically accessible world of "all after the "Big Bang" therefore namely doubling it transcendentally. So, the myth of the Bing Bang is due to the logical and conceptual misleading shared by all non-philosophical cognition (including physics, cosmology, astronomy, etc.) for the totality to be thought in the same way as anything within it, and so necessarily, being only a true part of it.

Thus, the Big Bang is misunderstood by common sense counting scientific in virtue of the aforementioned confusion therefore as a usual beginning of whatever being empirically accessible: the period of time "before the beginning" does make sense to the latter, but does not to the former. Consequently and particularly, the problem of what happened "before the Big Bang" is wrong in 
definition also messing the Big Bang with any empirical beginning studied by science experimentally. That confusion is due to the omnipresent identifying of all physics with empirical and experimental science therefore excluding and even "excommunicating and anathematizing" metaphysics from the "orthodox physics" at the cost of ridiculous beliefs in the "Big Bang" and others.

Time arrow accompanied with the understanding of the Big Bang in terms of the totality as a transcendental boundary implies the present moment of time as its dual counterpart. Speaking loosely, the Big Bang is the same as the present moment after the time arrow has been reversed, and vice versa as well. The "age of the universe", i.e. the period from the Big Bang until now, is due to the confusion of two concepts of time (which is not inconsistent for quantum mechanics does it very well): the one means the time arrow, e.g. as in thermodynamics, the other does not, e.g. as in special or general relativity. If both are adopted as in quantum mechanics, this generates the quantity "age of the universe" able to reconcile and equate those different concepts of time.

The visualization and corollaries from the identification of the Big Bang and the present moment as the same transcendental boundary under the condition of time arrow is much less investigated, and thus, much more interesting:

One means Minkowski space as a quadruplet (Cone,iCone,-Cone,-iCone) where " $i$ " is the imaginary unit, and "Cone" is an infinite 3-cone chosen so that Minkowski space is tautologically that quadruplet. Then, the usual light cone of special relativity is the surface of the pair (Cone,-Cone) coinciding with that of (iCone, -iCone).

The transcendental consideration meant here implies the identification (Cone $\equiv-$ Cone), or (iCone $\equiv-$ iCone) after the reversed time arrow (special relativity would be to mean the latter case). Both identifications can be identified with each other in turn if and only if both coinciding tops of each of them are identified as well: the name of the top in the former case is the "Big Bang", and that in the latter case is the "present moment".

In the "language of the totality", there exists a single cone, "Cone", which is a natural wellordering of the empirical Euclidean space. However, in the empirical language of physics valid only to any true part of the totality but not to the totality itself, the same cone is observed only quadrupled, i.e. only as the above quadruplet.

This can be represented by the "law of teleportation", according to which any qubit is omnipresent, but needs two additional bits of classical information (therefore travelling subluminally) to be restored exhaustively in a certain space-time point (where Bob receives instantly Alice's qubit from anywhere in the universe). Then, the identical qubit is interpreted in the language of the totality, and thus, as omnipresent, but Bob as a usual empirical physicist needs still "four letters" (i.e. two bits), "1, $i,-1,-i$ " transmittable only subluminally to determine unambiguously in the class of Cone equivalence:

\section{(Cone, iCone,-Cone,-iCone).}

Particularly, the "four letter theorem" (Penchev 2020 August 25; 2020 July 17) can be reconfirmed exceptionally concisely at the cost of the unification of physics and metaphysics as here.

There exists a shocking counterintuitive corollary (or even scandalous for the scientific common sense) after the identification of the Big Bang \& present moment. The age of the universe will be different in any space-time point of the universe for it depends explicitly on the space-time coordinates of the chosen point. Meaning furthermore consideration out of the present scope, one can suggest even that the "Standard model" valid in that space-time point depends implicitly on its coordinates. 
One might use those conclusions as a proof "ad absurdum" that metaphysics (at least the "totality") is inacceptable in physics. On the contrary, one can believe that they are correct and can be corroborated experimentally as well as that the totality implies directly observable phenomena such "dark matter" or "dark energy", which can be deduced formally and logically as the present paper tries to show.

(3) CPT-invariance as an invariance relevant to the "physical and mathematical transcendentalism":

CPT-invariance offers still one example (even especially instructive) of how physical and mathematical transcendentalism presences implicitly, entered "by stealth through the backdoor" in quantum mechanics and the foundations of physics.

Using again the above quadruplet notations, CPT-invariance means something turning out to be almost obvious: if both "P" ("space" with "letters" of the single bit: " 1 " and " $i$ ") and "T" ("time" with "letters" of the single bit: " 1 " and " -1 ") are the only necessary bits as the "law of teleportation" or the "four letter theorem" state, any additional bit e.g. such as "C" ("charge") would be redundant or able to replace each of "P" and " $T$ " bits as one of the necessary two independent bits among all the three. Thus, CPT-invariance means just this, and it is almost trivial once the present context of physical and mathematical transcendentalism has been adopted.

The following physical and philosophical interpretation of "charge" is relevant thus. The quantity of charge would have only two possible values (i.e. a single bit "C") notable as "externality" and "internality" of the totality in both sense and meaning of physical and mathematical transcendentalism. Then, the mysterious for a philosopher CPT-invariance can be translated as the following tautology:

If one changes all the tree bits " $\mathrm{C}$ ", "P", and " $\mathrm{T}$ " to the corresponding opposite letter (regardless of the sequence) in each of them, nothing will change. For example, the externality of the Minkowski real domain is the Minkowski imaginary domain (the space of special relativity), and the former needs the time arrow to be reversed. Now, one can see easily how physical and mathematical transcendentalism has entered special relativity and the foundations of physics "by stealth through the backdoor" formulating the following two statements:

1. The supraliminal ("real") domain of Minkowski space is the "externality of the totality" if the subluminal ("imaginary") domain of it is the "internality of the totality".

2. Physical and mathematical transcendentalism requires for the "externality of the totality" in the former statement to be representable equivalently in the corresponding "internality of the totality" again there.

The former statement means Minkowski space as the adequate mathematical formalism of special relativity, and the latter, a latent interpretation of general relativity, explicated here: Penchev 2013. Following that interpretation of general relativity in the present context, the interaction of gravity appears to compensate the violated CPT-invariance (being valid properly after the "flat" quantum mechanics) by curving space-time (therefore transforming both "P" and " $\mathrm{T}$ " bits into two corresponding independent qubits), and thus, restoring the broken symmetry by still one, but compensation violation (namely, the curving of space-time).

One can infer the same result by equating CPT-invariance and the "transcendental invariance" researched in detail in a previous paper (Penchev 2020 October 5) and utilized here again. That equating means CPT-invariance by the identification of the local and global space by the Standard model as the separable complex Hilbert space of quantum mechanics. Then, transcendental invariance complements still one equating: that of the separable complex Hilbert space of quantum mechanics 
to the pseudo-Riemannian space of general relativity under the "transcendental condition" (apropos, deducible form the postulation of the separable complex Hilbert space as the adequate mathematical formalism of quantum mechanics, and thus, consistent to CPT-invariance). After composing a single equating from both, the searched result is acquired.

The equation of the two invariance invariances to each other (CPT and transcendental) and even the equation of quantum gravity to general-relativity invariance following from the former implies an important conclusion verifiable experimentally and confirmed by all experiments until now:

Antigravity does not exist. An eventual discovery of any phenomenon explainable only by antigravity would mean that transcendental invariance would be a wrong postulate to physical reality.

On the one hand, it is the consideration that physical and mathematical transcendentalism implies for antigravity not to exist.

On the other hand, the same fallibility is not properly metaphysical statement in the sense of Popper and unlike the classical philosophical transcendentalism, from which originates. It only postulates the totality as fundamentally opposed to any empirical entity, and thus out of the scope and scale of experimental science. The experimental science itself reconfirms the same opposition therefore rejecting any metaphysical statement (even only alleged wrongly to be) in physics or experimental science. Still Occam's razor would cut it as redundant. Thus, our episteme seemed to be stable and reliable as far as two most important poles, experimental science with physics versus philosophy and metaphysics were separated both genuinely and definitively in all possible cognition. Thus, philosophical transcendentalism was closer to theology and religion postulating "God" analogically to the "totality" as fundamentally inaccessible empirically or experimentally, but only by internal experience whether belief or thought (as a rule, only with rare exceptions of "miracles") opposed to the external experience whether empirical or experimental.

The common contemporary understanding of mathematics is also too remote from the ideas and approach of classical German philosophy because of the following reason. The dominating axiomatic and deductive method for any mathematical theory to be built postulates though rather implicitly or on a meta-level explicitly that any axiom can be substituted by its negation (in a generalized sense) in the tuple of all axioms of the theory at issue and that modified theory will be true as its parent in virtue of the property "independence" of the initial tuple of axioms. That theory sharing this property is both consistent and complete but only relatively, to another mathematical theory, in which a model of the former is constructed and it can be proven as a theorem in the latter. Thus, a hierarchy of more and more fundamental models appears, and the Löwenheim-Skolem theorem proves that a very large and enough class of mathematical theories have consistent models in arithmetic, and thus, they are consistent and complete, but only in relation to it rather than absolutely.

Consequently, the problem whether arithmetic is consistent and complete in the final analysis seems to be irresolvable fundamentally since arithmetic occupies the lowest level ("level one") in the hierarchy, and thus, justifying all following ones starting traditionally from set theory as "level two". However much, much worse is the case. Gödel's theorems (1931) demonstrate this: the arithmetic itself is either inconsistent or incomplete to set theory, and thus, indirectly to all the class of theories meant in the Löwenheim-Skolem theorem. The gradual realization of that disastrous cognitive situation is well-known as the crisis in the foundations of mathematics in the beginning of the $20^{\text {th }}$ century: that is something is rotten in the state of ... mathematics. Many authors believe that the crisis is not overcome yet.

One dimension of the pathos of the present work is that the "rotten something" is in the state of cognition itself (i.e. the cognitive episteme of our epoch) and it only results in the foundation of 
mathematics as a particular case derivative from the "rotten" contemporary state of cognition. The crisis in the foundations of mathematics cannot be resolved only repairing the episteme since the cause of the crisis is to be removed. However, the eventual amendment of the foundations is only a background storyline in the discourse of the present paper (though main in others, e.g.: Penchev 2020 August 25): the plot here is about the analogical troubles in the foundations of physics in the beginning of the $21^{\text {st }}$ century when the humankind learnt that about $96 \%$ of the physical universe is "dark", presumably inaccessible fundamentally in the framework of the Standard model. The reformation of the underlying episteme would make the "dark" visible is what has to be proven explicitly. However the tool of the amendment, namely physical and mathematical transcendentalism resolves the crisis of mathematics as well and this cannot help but notice though rather only mentioned not in detail.

Physical and mathematical transcendentalism is able to resolve the crisis in mathematics being a non-experimental and rather non-empirical science (at least according to many authors, mathematicians or philosophers) so, but explicated only as a rough sketch ${ }^{2}$. That transcendentalism applied to arithmetic (let this be Peano arithmetic for unambiguous certainty) implies for it to be doubled as usual for transcendentalism. The uniform reason for transcendentalism to double refers to the totality though interpreted and named differently in each area of cognition. Especially to the foundations of mathematics, its hypostasis is the consistent completeness of mathematics therefore proved internally, i.e. absolutely and thoroughly within in and thus in a mathematical way.

Consequently, the totality (seen in mathematics as the mathematical totality of consistent completeness) applied to arithmetic would relate to the Gödel incompleteness as a relevant generalization of arithmetic by doubling able to overcome it. One considers the system of two complementary Peano arithmetic, on the one hand, and their identification as a single one (just as the transcendentalism requires) into single one, i.e. a usual Peano arithmetic, but interpreted nonstandardly in a way removing any well-ordering, but adding a cyclic structure instead of it. That generalized arithmetical structure called furthermore "Hilbert arithmetic" according to considerations which follow is only an interpretation of transcendentalism by the mediation of the concept of physical and mathematical transcendentalism applied to arithmetic granted as belonging to the foundations of mathematics.

One can shows rigorously and formally that Hilbert arithmetic (unlike Peano arithmetic) is consistent to both set theory and propositional logic therefore being consistent to the Gödel (1930) completeness theorems, but inconsistent to their 1931 counterpart for the substitution of Peano arithmetic by Hilbert arithmetic. Thus, the concept of Hilbert arithmetic is sufficient to overcome the crisis in the foundations of mathematics painlessly, i.e. in a conservatively generalizing way keeping all mathematics until now as it is as a true part (called Gödel mathematics) of the new generalized (called Hilbert) mathematics ${ }^{3}$. However, the same generalization admits the particular options for the Gödel and Hilbert mathematics to be as identical as subordinated as a true part of a whole accordingly, in virtue of the unique "transcendental identity" 4 .

\footnotetext{
${ }^{2}$ The same idea, again in the philosophical manner to "see the forest for the trees", is set out in detail in the study: Penchev 2020 August 25.

3 The concepts of Gödel and Hilbert mathematics and their relations to both Gödel completeness and incompleteness were introduced and discussed first on the following paper: Penchev 2010; however the idea was involved in: Penchev 2009.

${ }^{4}$ It can be exemplified and visualized by the identity of any infinite set to an infinite true subset under the condition of bijection.
} 
While the natural numbers of both complementary Peano arithmetics of Hilbert arithmetic are finite in the reference "frame" of the one Peano arithmetic itself, they will be seen as "transfinite natural numbers" (i.e. transfinite ordinal numbers are less, but not equal, than the least ordinal number among the ordinal numbers of all actual infinite sets) from the "reference frame" of the other, complementary "twin" (also a Peano arithmetic).

Involving the concept of Dedekind finiteness defining "finiteness" as to actually infinite sets in set theory, one can build a bijection of the separable complex Hilbert space of quantum mechanics and Hilbert arithmetic, and then, prove their isomorphism there crossing a "bridge" 5 of both physical and mathematical between the "riverside" of properly mathematical transcendentalism and the other "riverside" of properly physical transcendentalism.

Indeed, the separable complex Hilbert space (a.k.a. "Hilbert arithmetic") implies the completeness of quantum mechanics, which follows from the theorems of the absence of hidden variables in quantum mechanics (Neuman 1932; Kochen, Specker 1968).

Physical and mathematical transcendentalism is able to elucidate so the "dark substances" in physics being a science as experimental as empirical (and the pathway will be being developed and extended during this study):

The physical and mathematical concept of the totality identified as the universe is as mathematical and consistently complete as physical and implying "transcendental invariance". Then, the isomorphism of the separable complex Hilbert space of quantum mechanics and the pseudoRiemannian space of general relativity follows from it, and further, implies the mutual representability of entanglement as the tensor product of two (or more) non-orthogonal separable complex Hilbert spaces, on the one hand, and the gravitational field (in general relativity) as the relation of a tensor of energy-momenta and another tensor of space-time, determined unambiguously in the Einstein field equation.

Then, "dark matter" corresponds to the latter tensor, and "dark energy", to the former tensor after the equivalent "transcendental representation" of the entire entanglement of a certain state with all the universe (i.e. all other states of it) as an equivalent gravitational field under the condition of "Mach's principle" of Einstein (1918) in the following "paraphrase": the gravitation field of any source different from mass and energy (namely, such as quantum information, or "entanglement", directly) appears as an additional gravitational field of dark matter and dark energy, both confirmed experimentally very well.

The course of the paper will track that compose deduction via the intermediate stage of "quantuminformation conservation" in detail. However, the general picture, the "forest" is meant now: what premise implies what conclusion. Indeed, the conclusion of a physical phenomenon, such as dark matter and dark energy, follows from as a speculative and philosophical postulation such as that of the totality via the mediation of physical and philosophical transcendentalism. That is: a metaphysical premise implies a physical conclusion, and thus, the paper demonstrates that a class of both true and falsifiable deductions to physical conclusions from metaphysical premises is not empty. In other words, the intersection of metaphysics and metaphysics is meaningful, heuristic, and very, very fruitful as far as the "darkness" is the greatest problem of the contemporary physics.

Even more, the nonempty intersection of metaphysics and physics is equivalent to one of the most fundamental laws of nature: the conservation of quantum information.

\footnotetext{
${ }^{5}$ The idea of that "bridge" was set out first the book: Penchev 2009.
} 
Physical and mathematical transcendentalism, on the one hand, and philosophical transcendentalism can be related so within the composed deduction: the latter is the premise in turn following from the non-empirical postulate of the totality in philosophy, and the former is the formal pathway as mathematical and interpretable physically to many conclusions confirmable experimentally and empirically, or said otherwise, refutable experimentally and empirically.

What has to be emphasized is that physical and mathematical transcendentalism modifies philosophical transcendentalism essentially though the former originates and even can be seen as a formal and logical corollary from it.

Indeed, the philosophical common sense adopts transcendentalism as the most of philosophical doctrines, i.e. as those defined by Popper as "metaphysical" in the sense of "irrefutability" as formal, logical, and mathematical as experimentally or empirically. The standard philosophical doctrine is "immunized" against logical inconsistency not acknowledging the criterion of logical consistency as well as against any empirical and experimental refusal again not acknowledging it as the former. It originates from a certain doubtless philosophical "authority' such as Kant as to transcendentalism or Hegel as to dialectics. The corresponding doctrine is granted as true by many people, especially philosophers, and this is its confirmation, properly,

The revision of physical and mathematical transcendentalism in relation to the standard philosophical transcendentalism is that it accepts the criterion of refutability of both mathematics and physics and thus trying to prove its truth in the real dilemma of true versus false. This allows for it to deduce experimental and empirical corollaries therefore being testable.

Nonetheless, it continues to be not less a form of philosophical transcendentalism in virtue of realizing itself as a new "quantum" Pythagoreanism6.

That relation of physical and mathematical transcendentalism opposed to philosophical transcendentalism implies the following about the verifiability (fallibility) of the latter: it is still metaphysical in Popper's sense as far as physical and mathematical transcendentalism though adopted logical or experimental refutability is an absolutely independent philosophical conception. Thus, the eventual refusal of physical and mathematical transcendentalism would not touch the validity of philosophical transcendentalism relied on people's acknowledgment. Anyway, the absence of any refusal (and thus, indirect confirmation) of physical and mathematical transcendentalism would be also in favor of philosophical transcendentalism acquiring wider acknowledgement.

An example of the subtle difference between Hegel's dialectics (as closely related to Kant's transcendentalism) and physical and mathematical dialectics (as inferable from physical and mathematical transcendentalism) can be suggested by the expansion of the universe, very well confirmed experimentally.

One the one hand, the Hegelian (including Marxist) dialectics explains the self-movement of the totality by itself as definitive to it (and eventually consistent to philosophical transcendentalism). However, it considers the universe (in particular) rather as a metaphor than an interpretation of the totality. Like so, any as confirmations as refusals both experimental and empirical about the universe are not valid to the totality (since the link of metaphor is not logical).

\footnotetext{
${ }^{6}$ Though Platonism is met often among the mathematicians and rarely among the physicists, Pythagoreanism is almost absent among both groups. Pythagoreanism can be consider as an extreme, radical form of Platonism, in which the ideas are identified as "numbers". The quantum form adds the "complementarity" of "numbers" and "things". Though a part of the founders of quantum mechanics can be accepted as "quantum Platonists" (.e. adding the complementarity of "ideas" and "things"), the conception of quantum neo-Pythagoreanism is not available among them.
} 
On the contrary, the physical and mathematical transcendentalism seems to be "childish" and "naïve": it adopts the above metaphor as an interpretation, even more, formal and logical interpretation. This is an obvious "mess" and "confusion" of formal logic with the do-called dialectic logic from the viewpoint of the orthodox dialecticians. However, this is a consistent, formal and logical agreement of physical and mathematical dialectics with the theory of information generalized as quantum information as the most fundamental and omnipresent substance in nature from the opposing viewpoint of the present paper.

Hence, the physical and empirical confirmations or refusal referring to the universe reflect back in a precise logical meaning to what is interpreted as the universe then, i.e. to the totality of dialectics therefore transforming dialectics in a falsifiable theory as the usual ones in science and unlike the most of philosophical doctrines. In other words, physical and mathematical transcendentalism does not search for its confirmation in people's acceptance, but in experimental and empirical facts (in face of which both people and "gods would be to be silent").

On this background, one can consider the expansion of the universe (reversely projected in the hypothetical beginning of the Bing Bang and discussed already above) as an interpretation of the selfmovement of the universe by itself in a Hegelian manner. Physical and mathematical transcendentalism implies that self-movement (in the framework of physical and mathematical dialectics) as follows:

The doubling of anything, definitively owing to the totality, is as equivalent as indistinguishable from the Hegelian philosophical motion, or "general change", i.e. the same. A physical interpretation of it is mechanical motion. Then, that physical interpretation together with the other one about the totality as the universe, imply the observable self-movement of the universe as the "expansion of the universe". The same deduction implies still one falsifiable forecast: the opposed "shrinking of the universe" is impossible, and thus, it cannot be observed ever. Here is why:

The transformation of the transcendent in the transcendental is only in the one direction (namely this) just meant in time arrow. The hypothetical observation under the condition of the reversed time arrow would register that "shrinking of the universe". However, this is a "mistake in definition" because of the contradiction in the following point:

The "observation" contradicts the "reverse time arrow". Indeed, the observation by itself generates an asymmetry between who (what) observes and what is observed meant physically and empirically in time arrow. So, the reversed time arrow reverses also that asymmetry into the opposed one or logically into the non-observation directly contradicting the presupposed observation not less imposed by the postulate of empirical and experimental science. Particularly, the "shrinking of the universe" (apropos, discussed absolutely seriously by the contemporary physics and cosmology for their ignorance in metaphysics) is impossible fundamentally and in principle (of course, under the refutable condition of physical and mathematical transcendentalism).

(4) Dirac's "magnetic monopole" does not exist

Unlike philosophical transcendentalism until now, physical and mathematical transcendentalism implies corollaries verifiable experimentally. The main objectivity of the paper, the deductive pathway from physical and mathematical transcendentalism to dark matter and dark energy is among them. The text and context suggest others as well.

One of them, the necessary inexistence of Dirac's monopole will be considered now just as an example for mathematical and physical transcendentalism is not a metaphysical conception (in Popper's sense), therefore not allowing for any refusal. Indeed, if there exists the magnetic monopole, it would be wrong. 
That demonstration requires the deduction of the nonexistence of the monopole from physical and mathematical transcendentalism to be inferred expressively:

Dirac (1931)'s conjecture of magnetic monopole means separated magnetic charges similar to the separated electric charges furthermore invested as in Maxwell's electrodynamics as in quantum electrodynamic. His purely theoretical reason is that the existence of that "monopole" is consistent to quantum electrodynamics though, on the contrary, its nonexistence is postulated as one from the four laws of electromagnetic field in Maxwell's theory. However, the nonexistence of magnetic monopole is also consistent to quantum electrodynamics. In other words, it can be admitted, but cannot be forecast from quantum electrodynamics.

Physical and mathematical transcendentalism is able to interpret the magnetic monopole as follows. After the bit of charge ("C" in the consideration of CPT-invariance above) is meant by two "letters" corresponding to the "externality of the totality" and the "internality of the totality", the transcendental principle implies the necessary option for the separated polar electric charges to be able to be together as the same, fundamentally and inseparably. That option of transcendental counterpart or hypostasis of any pair of two polar electric charges ${ }^{7}$ is a corresponding magnetic dipole therefore excluding any magnetic monopole. Speaking loosely and rather metaphorically, Dirac's monopole exists only by the transcendent counterpart, i.e. in the form of the pair polar electric charges, and by itself, only in the form of transcendental twin, being a dipole always in virtue of physical and mathematical transcendentalism.

The concept of electromagnetic field in both Maxwell and quantum electrodynamics unifies electrical monopole charges and magnetic dipole charges as inseparable in any point of space or space-time. However, quantum electrodynamics as any quantum theory admits additionally the option of quantum leap, and thus, of separability in relation to any quantum entities of the same name, particularly, those of the same magnetic dipole.

The permission of quantum mechanics to Dirac's conjecture is due to the same class of reasons as that to the standard quantum gravity meaning it as still one fundamental interaction analogical to the rest three in the Standard model.

On the contrary physical and mathematical transcendentalism rejects both as well as the general kind of permission, including particularly those to each of them. It imposes additional restrictions on quantum theories, the violation of which would refuse it.

The transcendental viewpoint to electromagnetic field furthermore allows for an interpretation (or justification) of the postulate in special relativity for the boundary of light speed in a vacuum therefore being a fundamental constant. That postulate is justified usually only as a very well corroborated experimental fact, but not as deducible from other principles.

However, physical and mathematical transcendentalism implies that postulate in special relativity, indeed rather partly in a clear sense as follows. As Dirac thought and wrote, a vacuum can be considered as a medium in quantum superposition. One can involve further an observer in any point and decohering the vacuum starting from the point, in which an observer is meant.

That specific speed of decoherence featuring a vacuum is the fundamental constant of light speed in a vacuum furthermore postulated in special relativity and transferred then into both general relativity and quantum mechanics.

\footnotetext{
${ }^{7}$ They can be thought as "transcendent" to each other after the one belongs to the "externality of the totality", and the other, to the "internality of the totality".
} 
In terms of quantum mechanics, it can be visualized so: how $r$ from each other two space points should be so that their temporal simultaneity (in the sense and meaning of special relativity) can be established only by a quantum leap. Speaking only figuratively, this means what minimal space distance implies a quantum leap between its extremes to be simultaneous, i.e. that "causes" space discreteness, or as if space to be "broken". The process of decoherence represents just that space "break" only after which space-time has appeared since it does not make sense in relation to a coherent vacuum, but only to a decohered one.

The Big Bang conjecture means that space-time has appeared not later than it, and thus, it is available in advance for any real physical process. On the contrary, the present context implies that it appears constantly and always as a specific aspect of any physical process, and thus, it can be projected in a hypothetical beginning called the "Big Bang" only as a myth: the states of the universe in the "first seconds" and later after it are not real states but only mathematical properties of that projection proclaimed ostensibly to describe a "reality", namely that after the "Big Bang", as a mathematical and theoretical model. In fact, that model is the reality itself, and the Big Bang is the mythological projection onto our cognitive and cultural episteme postulating for any mathematical model to be only secondary in relation to a certain reality, which only describes, but itself cannot be in definition. In other words, quantum mechanics needs its model to coincide with reality by itself, but this is so "unbearable" of the collapsing cultural prejudice and cognitive episteme.

Once one has adopted sincerely what quantum mechanics heralded unambiguously a long time ago, the Bing Bang vanishes in thin air, but who dare do that finds himself or herself out of our age and its episteme ${ }^{9}$ ?

How does space-time appears unambiguously in any physical processes once the fundamental constant of light speed in a vacuum has been introduced whether inferred or postulated as in special relativity?

That aspect of any physical process referring to the nascency of space-time is just electromagnetic field propagating in a vacuum with light speed. Physical and mathematical transcendentalism allows for the "birth" of space-time in any physical process to be visualized by concepts. Here is how:

Light speed is interpreted so. It means the ratio of the distance between two points of the appearing space, being inseparable (a unit of space) and the distance between the same two points being separable (a unit of time). As far as the light cone of special relativity (by means of Minkowski space) belongs to both subluminal superluminal area as their boundary, that ratio defines just the light cone.

\footnotetext{
${ }^{8}$ Einstein (1926) used the cited word in his famous letter to Max Born.

${ }^{9}$ Giordano Bruno and his tragic destiny can serve as an example of a man out of his epoch and its episteme though the epoch is other. Giordano Bruno's visitation and "acceptance" in Oxford are the subject of scientific articles and books [Yates 1939; Gatti 1989; McMullin 1986; Feingold 2004; Massa 1977; McNulty 1960; Pellegrini 1942]. Taking an occasion from them, he can be involved also as a character in the present discourse therefore personalizing the drama or conflict between the empirical and the philosophical (still more involving a neo-Pythagorean referring to the universe and its infinity and the "dialectics" of its maximum and minimum in a manner recollecting that of Nicolas of Cusa, as he did); for example, the multiple worlds in the universe as an outlook defended by him. The scholars of Oxford rejected it for the absence (then) of empirical confirmations rather than for its contradiction to the Bible and Church dogma or to Aristotle's doctrine. Well, that rejected worldview is common and the base of experimental research in science nowadays. Are we to justify or blame the scholars of Oxford? What is the responsibility for the unconditional rejection of ideas confirmable in the future? How should one relate the scientific theory falsifiable empirically (or experimentally) and the philosophical doctrine whether accepted or not (e.g. as Giordano Bruno's)?
} 
Then, the magnetic dipole (therefore excluding any magnetic monopole) corresponds to the former and means the appearing space as appeared already, and the pair of opposite electric monopoles, to the lather and means the appearing space as not appeared yet. Consequently, it represents the frontier of the appearing space, which only is able to be both appeared already and not appeared yet without any contradiction, i.e. just appearing.

Though Dirac's monopole cannot be observed since it does not exist fundamentally, either purely magnetic phenomena or purely electric phenomena can be observed standalone as this is well-known. The explanation is simple:

The facts of electromagnetic induction show that only moving whether magnetic dipoles or electric monopoles can be associated with electromagnetic field. The motion is a physical process and therefore accompanied with generating space-time by electromagnetic field. Indeed, the motion means always a new point to be applied by a hypothetical "observer" and a new process of decohernce to start from that point therefore implying electromagnetic field. The state at rest means the same hypothetical observer in a series of previous moments, and thus, an implicit previous process of decoherence generated the space-time as available in advance. In that derived and inherited spacetime, i.e. existing since a time, the corresponding counterpart is able to vanish in virtue of neutralization ${ }^{10}$.

The same properly physical aspect for a vacuum to decohere can be interpreted by physical and mathematical transcendentalism just as both physical and mathematical at the same time. Then, the magnetic dipole would correspond to the transcendental unity of the transcendental boundary between the immanent and transcendent, particularly as a pair of space points, and the pair of electric poles, of the transcendental opposition of the same. The former (i.e. that "of unity") and latter (i.e. that "of opposition") sides are featured from each other as different qualities, therefore avoiding the contradiction, or as different physical quantities, accordingly distance and time. The correspondence is unambiguous. Just the quantity of time should be related to "opposition" for it means a wellordering in definition, and thus, the well-ordering "theorem" and the axiom of choice, and further, a discrete leap (what should be able to feature the opposition of the immanent and transcendent) in the final analysis. On the contrary, the quantity of distance should be related to "unity" since it presupposes the space as available already (unlike the opposition, to which it has not appeared yet). The fundamental constant ratio of the two quantities in turn holds on both sides of the transcendental boundary linked to each other inherently ${ }^{11}$.

The step to the properly mathematical interpretation of the fundamental constant of light speed in a vacuum is obvious already. The class of equivalence of all constants of the same kind (i.e. the variable, to which "our constant of light" is only a certain value) can be defined mathematically thoroughly: it describes how any well-ordering (what the physical quantity of time is also) can appear from any class (particularly by a set by the axiom of choice). In other words, the light constant seen mathematically rules the process of ordering to be unambiguous, or "without mistakes". Thus, it need

\footnotetext{
${ }^{10}$ Neutralization means for electric monopoles to constitute dipoles disoriented in general or to annihilate, and for magnetic dipoles to be disoriented so that the magnetic component of electromagnetic field to be zero as to the ensemble of magnetic dipoles (respectively, as to electric dipoles). The process of neutralization is possible only in an also available space-time, but not in an appearing one, i.e. in any electromagnetic field, in which both components are available in definition.

${ }^{11}$ Speaking quite figuratively, the light speed in a vacuum (or electromagnetic field) "delivers" the space (as a "baby") from time, or the philosophical "being" (Heidegger's "Sein") from time or the philosophical "time" (Heidegger's “Zeit”).
} 
be a constant ratio of how (whether "much" or "many") of what is nor ordered is equal to a unit of the ordered (what the usual arithmetical " 1 " is almost always) therefore regulating the arithmetical models of anything being mathematical to be isomorphic to the modelled entity, and thus, implicitly meant in the Löwenheim-Skolem theorem or Skolem's "paradox" in particular.

The reverse reflection from the purely mathematical interpretation back to physical and mathematical transcendentalism implies the understanding of the light constant to supply for the transcendental physical image of the transcendent entity after the totality to be correct, adequate, or isomorphic mathematically.

(5) The equivalence of the Hamiltonian and Lagrangian formulations of both classical and quantum mechanics

Though Lagrangian formulation is historically first ${ }^{12}$, Hamilton demonstrated that classical mechanics can be reformulated equivalently under the condition for the (generalized) space coordinates and corresponding components of momentum (three momenta) to be two kinds of independent variables. That is totally six therefore constituting phase space and admitting implicitly a thermodynamic reinterpretation of classical mechanics. The quantity of time is not necessary though it can be added consistently.

The two interpretations are absolutely equivalent and the Hamiltonian one is rather auxiliary helping technically for practical problems, and curious or even exotic for common sense. Its philosophical meaning is impenetrable from the absolute and unambiguous determinism of classical mechanics.

Quantum mechanics was formulated initially in a Hamiltonian manner for it seemed to be convincing intuitively. For example, the status of time was the same, the phase space reformulation was attractive, and Bohr's complementarity directly interpretable, etc.

The separable complex Hilbert space, however in fact, as the space of wave functions, equates the Hamiltonian and Lagrangian interpretations of quantum mechanics at the cost of involving the nonclassical entity of wave function (or quantum information in contemporary terms). This can be illustrated elementarily by the Schrödinger equation, in which the Hamiltonian formulation both implies and follows from the Lagrangian one. Here is how:

The Schrödinger equation is formulated in a way to unify features of both formulation consistently. Its formulation about the temporal evolution of a quantum system will be meant for certainty. On the one hand, it involves time explicitly as the Lagrangian formulation does, and on the other hand, time and space are divided disjunctively: the one is in the left side (conventionally) and the other is in the right side (conventionally). Hence, one acquires instantly its "Hamiltonian form": the "momentum part" in the left side is equated to the "space part" in the "right side". And vice versa as well: the "Hamilton" form is equated to the in the initial Lagrangian one by the reverse trivial transformation.

This can be realized by Bohr's postulate about what quantum mechanics studies, namely: only systems of quantum entities and the apparatus macroscopic and described in terms of classical mechanics by the readings of the latter. This means quantum mechanics can be defined only as a conservative generalization of classical mechanics. If one rejects classical mechanics, quantum mechanics is also rejected necessarily. In other words, quantum mechanics contradicts only our prejudice of common sense, but not classical mechanics for this is impossible in definition.

\footnotetext{
${ }^{12}$ The initial, "intuitive" Newtonian formulation precedes both Lahgrangian and Hamiltonian ones.
} 
Then, the Schrödinger equation interpreted in a Lagrangian manner follows exactly Bohr's postulate equating the temporal evolution of the quantum system (in the left side as usual) and thus valid only to the readings of the apparatus, to which "time" make sense, on the one hand, and the system of the quantum entity and the apparatus (in the right side as usual), to which "time" is not valid for Pauli's "time is only a number" (i.e. not an operator); otherwise, it would not be able to represent adequately the investigated quantum system. To succeed and manage its mission, the Schrödinger equation involves a fundamental new kind of element both physical and mathematical simultaneously: wave function (or the substance of quantum information), which is that is equated properly. The last fact predetermines the equivalent Hamiltonian (as if) reading of the Schrödinger equation:

This reading means: the wave function of the quantum system is the same as what is extracted from the readings of the apparatus for quantum information (wave function) is conserved. Furthermore, the quantum system and the macroscopic apparatus are complementary in Bohr's sense even only to be consistent to time available in the apparatus, but not in the quantum system. Thus, the one side (never mind which of both) of the equation seen a Hamiltonian manner depends only of the one of the conjunctive pair of independent variables; and the other side respectively.

The instruction is: the Lagrangian time implies the Hamiltonian independence of space coordinates and momentum components from each other as well as vice versa. This condition can be interpreted "transcendentally" (i.e. in terms of the "transcendental condition") as necessary and sufficient for the equivalence of the two formulations of quantum mechanics itself in terms of wave functions. Indeed:

The explicitly temporal (and thus as if) Lagrangian formulation of the Schrödinger equation corresponds to the properly transcendental consideration of the totality, after which the "externality of the totality" is granted within the "internality of the totality" under the necessary condition of wellordering physically represented by time arrow therefore involving necessarily in turn the time itself.

On the contrary, the (as if) Hamiltonian formulation, explicitly divided into two kinds of independent variables, corresponds to the immanent - transcendent doubling of the totality by itself. The one half variables would be to be qualified as "immanent" (conventionally), and the other half, as "transcendent" (in convention), after which they have to be equated just as the Schrödinger equation does in relation of both its sides.

In other words, the equivalence of the Hamiltonian and Lagrangian formulations implies the transcendental condition applied to quantum mechanics (i.e. it is necessary and sufficient in its scope in virtue of the cited argument only).

Thus, the Schrödinger equation (being in terms of wave functions) reconciles both formulations to each other as equivalent. Further, it implies a proper Lagrangian formulation of quantum mechanics, the opposite extreme counterpart of the proper Hamiltonian mechanics, but appeared historically first. The former even is preferred rather in the contemporary quantum mechanics in a series of technical advantages.

(6) The concept of information both physical and mathematical and thus fundamental in physical and mathematical transcendentalism

The equivalent interpretations of the Schrödinger equation once in a Lagrangian manner, twice in a Hamiltonian manner, offers an occasion to be realized the reconciliation of physical and 
mathematical dialectics to formal logic (unlike philosophical dialectic ${ }^{13}$ ) embedded in the conception of information and its unit, an elementary choice. That is the pathway:

One suggest a fundamental, formal and logical tautology being the definitive proposition of the totality (in terms of formal logic, properly). This tautology can be extracting as underlying the isomorphism of the Hamiltonian and Lagrangian interpretations of the Schrödinger equation (as it will be shown soon further): It is:

$$
\text { "A is true" } \Leftrightarrow\{[(\mathrm{A} \Rightarrow \mathrm{B}) \Leftrightarrow(\mathrm{A} \Rightarrow \neg \mathrm{B})] \Leftrightarrow[(\mathrm{A} \Leftrightarrow \mathrm{A}) \Leftrightarrow(\mathrm{B} \Leftrightarrow \neg \mathrm{B})]\}
$$

The notations " $\Leftrightarrow ", " \nLeftarrow ", " \Rightarrow$ ", and "ᄀ" mean correspondingly logical equivalence, inequality, implication, and negation; a part of the brackets is added only to facilitate the reading by humans.

Then, that logical tautology is interpreted once (6.1) as a formal and logical definition of the totality, twice (6.2) by a bit of information, and trice (6.3), as underlying the isomorphism of the Hamiltonian and Lagrangian "reading" of the Schrödinger equation.

(6.1) "A is true" is interpreted as "A is true definitively" or "A is true" is a definition of A", i.e. one means the proposition A which cannot help but be true: it is true tautologically. It is a logical tautology in the final analysis, e.g. such as "The true proposition is true". If one involves the distinction of the synthetic and analytic, relevant to Kant's transcendentalism, it is analytically true necessarily.

Furthermore, being a proposition like any other, it would be to define a relevant class not empty only in general. For example, that kind of class is meant in the set theory as the universal set ${ }^{14}$. The essence consists in the distinction between the proposition and the corresponding class (set) though linked unambiguously. The totality is the class (set) so ascribed to tautology. Thus "A is true" is able to mean the totality defined logically and it means it indeed under the condition of the above interpretation.

In terms of the opposition "synthetic truth - analytic truth", those link and distinction serve to transfer an analytic true statement such as one in logic and mathematics (e.g. referring to any physical model of any physical theory) into its interpretation literally coinciding with it, but being a falsifiable by experience or experiments synthetic true statement (e.g. referring to the physical theory itself meant by the mathematical model at issue).

One can notice that a logical doubling appears following the general scheme of physical and mathematical transcendentalism (by the example of logical transcendentalism in the framework of physical and mathematical transcendentalism). Transcendentalism merges ontology and epistemology into the same: ontology means the double and doubling as a single one, and epistemology the same as just doubled or doubling. Then, transcendentalism is that single one doubling and doubled as both ontology and epistemology.

Furthermore, the scheme is the same in both level and meta-level therefore transforming any hierarchy of levels into a single whole what the totality is in definition. Indeed one will be able to see a little further that the scheme satisfies the above fundamental, formal and logical tautology, that of the totality.

\footnotetext{
${ }^{13}$ Philosophical dialectics has given, gives and will give an impetus for new and new non-classical logics, particularly paraconsistent. This direction tends to reformate logic to be consistent to philosophical dialectics. Maybe, "Mohamed to go to mountain" is better: philosophical dialectics to be reformatted as the falsifiable physical and mathematical dialectic just a way to be consistent to the classical "formal logic". The present paper is moved by that motivation.

${ }^{14}$ Meaning of Russell's paradox about the universal set from the viewpoint of physical and mathematical transcendentalism will be discussed in detail in a subsection (8) of the present section.
} 
Summarizing, the totality is introduced logically in Kant's track, or in his manner to answer his own question:

How is synthetic a priori knowledge possible?

The totality is what makes possible that knowledge and features philosophical cognition. As being analytic, it can be granted as metaphysic in Popper's sense, not being able to be falsified. However, transcendentalism following its own scheme generates by itself its falsifiable synthetic counterpart, physical and mathematical transcendentalism and it is what is meant during the paper properly as unifying as distinguishing physics and metaphysics.

(6.2) One can interpret the "right side" of the fundamental tautology as the equivalent logical description of a bit of information; namely:

$$
\{[(\mathrm{A} \Rightarrow \mathrm{B}) \Leftrightarrow(\mathrm{A} \Rightarrow \neg \mathrm{B})] \Leftrightarrow[(\mathrm{A} \Leftrightarrow \mathrm{A}) \Leftrightarrow(\mathrm{B} \Leftrightarrow \neg \mathrm{B})]\}
$$

Let one mean the description of a bit of information by a cell of the tape of Turing machine. That exemplification is involved only for the visualization of the interpretation to be more distinguishable in both logical operations and physical actions. Then:

"A $\Rightarrow$ B" means the 'record of " 1 " in a cell'.

" $\mathrm{A} \Rightarrow \neg \mathrm{B}$ " means the "record of " 0 " in a cell'.

"A $\Leftrightarrow A$ " means that "the cell is the same regardless of whether " 0 " or " 1 " will be recorded (or not) in it'.

" $\mathrm{B} \Leftrightarrow \neg \mathrm{B}$ " means that the "records of either " 0 " or " 1 " are disjunctive to each other, or the "excluded middle".

Then the "right side" of the tautology interpreted as an exhaustive logical description of a bit of information (in turn interpreted as a tape cell of Turing machine) would mean: the record of either " 0 " or " 1 " in a cell is equivalent to the equivalence of an empty sell and the same sell, in which either " 0 " or " 1 " are recorded; and this is the exhaustive logical description of a tape cell of Turing machine or a bit of information.

The visualization emphasizes the equation of the physical (the record of either " 0 " or " 1 "), i.e. being temporal or continuous, and the logical (the equivalence of the empty sell by itself to it, being in a "recorded state") i.e. being non-temporal and discrete.

Though bulkier, the visualization of a bit of information as an elementary choice between two equally probable alternatives interprets that logical expression in an isomorphic way.

Then, the tautology can be written concisely by the verified logical description of a bit of information:

\section{"A is true" $\Leftrightarrow$ 'one bit of information'}

Well, this seems to be trivial and obvious as far as either "A is true" or "A is false": just one bit of information.

(6.3) One can realizes particularly the tautology as follows:

"A is true": only A is meant where " $\mathrm{A}$ " is the system of the investigated quantum entity " $\mathrm{B}$ " (conventionally) and the apparatus " $\neg \mathrm{B}$ " (in convention).

"One bit of information": "A" as "A" is the system of the investigated quantum entity "B" (conventionally) and the apparatus " $\neg \mathrm{B}$ " (in convention). Then one can check the meaning of both Hamiltonian and Lagrange "readings" of the Schrödinger equation in terms of the "logical description of a bit of information".

First, the Lagrangian "reading" means the isomorphism of the "record" of the system " $A$ " whether in the entity " $\mathrm{B}$ " or in the apparatus " $\neg \mathrm{B}$ " and vice versa (i.e., and the alternative complementary "record" in the corresponding counterpart whether " $\neg \mathrm{B}$ " or "B"). However, the historical and 
traditional structure of the Schrödinger equation explicates expressively that isomorphism only with accordance to Bohr's postulate about what quantum mechanics studies. That is: it equates the record of "A" (conventionally, in the right side of the equation where time is not variable) onto " $\neg \mathrm{B}$ " (conventionally, in the left side where time is variable, on the contrary).

Second, the Hamiltonian "reading" means the isomorphism of "A" by itself (interpretable as the quantum superposition of " $B$ " and " $\neg B$ " or as the "empty cell" of a bit of information) and the complementarity of " $\mathrm{B}$ " and " $\neg \mathrm{B}$ ". Just as in the case of the Lagrangian reading, the historical and traditional structure of the Schrödinger equation explicates expressively that isomorphism only in accordance to the classical Cartesian dichotomy of the "object" of the entity and the "subject" of the apparatus (before that predetermining the kind of the Hamiltonian "reading" of classical mechanics where that kind is not only consistent as well). That is: it equates the state of the entity by itself to that of the apparatus as another quantum system by itself analogically ${ }^{15}$.

Then, the equivalence of the Lagrangian and Hamiltonian "readings" interpreted correspondingly as the "left and right side" (as ordered conventionally in the logical expression of the tautology above) in the equivalence of a "bit of information" described logically can be interpreted quite reasonably according to the (6.1) transcendental interpretation of the tautology as the system being an inseparable or "contextual" whole therefore exemplifies the totality and inherently to the featuring holism of quantum mechanics.

One can find the same "single bit of information" still in the separable complex Hilbert space of quantum mechanics: indeed, the choice between the two dual twins of it is just one bit information, furthermore interpretable in accordance to as Hamiltonian "reading" standalone as the equivalence of the Lagrangian and Hamiltonian "readings". This is obvious as far as the discussed tautology is the necessary condition the mathematical formalism of quantum mechanics.

What remains (6.4) to be done is the three interpretations (6.1., 6.2, and 6.3) to be joint and unified in a single whole: a formal and logical proof of the model of the universe within a single quantum, furthermore relevant to cyclic ontology, in which the biggest, the universe is the same as the smallest, a quantum in the manner of Nicolaus Cusanus or Giordano Bruno. Explicitly:

The universe is interpreted by "6.1", a bit of information, by " 6.2 ", and " 6.3 " implies the conservation of quantum information (in detail in Section VII further), which means both duality and identity of the quantity of physical action and that of quantum information, proportional to each other by the crucial mediation of the Planck constant. Its magnitude does not matter, only its existence as a constant or even as a variable is necessary for the ontology named as "the universe within a quantum": any nonzero (not infinitesimally small as well) magnitude of the Planck constant determines how a quantum is "big", but not the essential quality of its discreteness.

Concisely: as a logical tautology, the corresponding interpretation of it is true as well. The interpretation is: the universe is (logically equivalent to) a bit of information, which in turn is (logically equivalent to) a quantum in virtue of quantum conservation inferable from " 6.3 ". The only consistent way to be equated the universe (not possessing any externality as an exemplification of the totality) and a quantum (the internality of which, oppositely, is prohibited as a natural law according to the meaning of the Planck constant), furthermore in accordance to physical and mathematical

${ }^{15}$ By the way: that Hamiltonian reading of the Schrödinger equation implies, and even, makes obvious the conservation of quantum information. 
transcendentalism, is by the coincidence of the upper boundary of the former with the upper boundary of the latter. This generates cyclic ontology also for the availability of quanta within the universe ${ }^{16}$.

(7) The consistent completeness of mathematics as the mathematical totality:

The mathematical totality would mean that mathematics (or at least a certain part of it) which can be considered in a way to contain all the non-mathematical within itself. Thus, mathematics would avow to be the most fundamental science, a claim shared by philosophy and physics though in different ways. Their assertions to be most universal can be distinguished from each other by the conception of truth for each of them since the "profession to be universal" depends crucially of how truth is understood and heralded ${ }^{17}$.

In fact, mathematics shares two different conceptions of truth, however they can be unifies into a single one by physical and mathematical transcendentalism therefore interpreted and modified by it:

The one is related to that of experimental and empirical science and generalized Western philosophy in the conception of truth as adequateness: the mathematical models should be adequate to the corresponding theories, to which they refer and by them, to reality by itself presupposed as external to mathematics. Thus, that be called the "external criterion of truth in mathematics".

However, physical and mathematical transcendentalism transforms it involving the mathematical totality: reality can be represented adequately first by a relevant scientific theory, furthermore empirical and experimental, and then, mathematized by a formal model ${ }^{18}$ : the correspondence of reality and model is also mathematical, i.e. a mapping, fundamentally unambiguous: a bijection, hence, generating a properly mathematical interpretation of transcendental doubling, namely the class of all bijections of reality and the mathematical totality, i.e. a new contemporary form of Pythagoreanism.

A bit of information, being equivalent logically to the totality according to the "fundamental, formal and logical tautology" in Subsection 6 above, in turn is isomorphic to an elementary bijection of that transcendental and mathematical doubling ${ }^{19}$. Consequently, mathematical transcendentalism as the one complementary "half" of mathematical and physical transcendentalism, is a direct trivial corollary from "logical transcendentalism" (once it is considered as above) and even only renaming of logical notions by mathematical ones.

The other conception of truth in mathematics is internal, or properly mathematical. It needs a proof for the consistent completeness of each formal and mathematical theory (i.e. an extended

\footnotetext{
${ }^{16}$ The transformation of one universe (i.e. one quantum) into many quanta within it is also explainable by the transition through the transcendental barrier separating disjunctively the universe from one (any) quantum: the universe as a single quantum means the class of equivalence of all quanta within it only where they can be distinguished or "named" with the generalized coordinates of the point of the universe where they are (whatever the mathematical space to which they belong is).

${ }^{17}$ For example, Christianity professes universality not less than philosophy, physics, and mathematics, but understandanding it according to the Bible and the said by Jesus (John 18:37, for example). Then, Pontius Pilate's "Quid est veritas?" follows (John 18:38). Well, philosophy, physics, and mathematics answer Pilate's question differently being differently but all the three, dissimilarly to Christianity or any other religion. So, each of them believes to be universal, but understands "What is universality" by its own answer of "What is truth?"

${ }^{18}$ Thus, one can introduce the principle "All science is rigorous science": an obvious allusion to Husserl. That principle states that any theory is mathematizable even constructively (Penchev 2020 June 29).

${ }^{19}$ Indeed, utilizing again the visualization of a bit of information by a tape cell of Turing machine, the elementary bijection itself corresponds to the sell by itself, the element of reality mapped by the bijection, to " 0 " (respectively, " 1 " as the symbol is conventional), and the element of the model mapped by the bijection, to "1" (respectively, "1").
} 
syllogism built axiomatically and deductively). The Lövenheim - Skolem theorem in (ZFC) set theory allows for (Peano) arithmetic to be proved as a universal standard, in which a relevant model of any formal and mathematical theory defined as above can be constructed in way the consistent completeness of the model implies the consistent completeness of the theory.

Thus, if one manages to prove consistent completeness of arithmetic or at least that of any possible model (within it) of mathematical theory the internal criterion of truth in mathematics would be verified. However. Gödel (1931) made do the opposite with (Peano) arithmetic, (ZFC) set theory, and (Principia Mathematica) logic. He proved that as arithmetic as any theory implying it is either incomplete or inconsistent to set theory. Physical and mathematical transcendentalism is able to overcome this therefore reaching the internal proof of the consistent completeness of mathematics by the relevant generalization of Peano arithmetic on the base of its principles namely doubling it as two complementary twins of Peano arithmetic. A concise sketch ${ }^{20}$ will be outlined a little further.

Before that, one can notice that the Lövenheim - Skolem theorem generates an internal and properly mathematical repetition of the external criterion of truth in mathematics, visually representable by the following analogy (which in the framework of physical and mathematical transcendentalism is much more than analogy, and even an isomorphism): what is arithmetic to mathematics is mathematics to reality. However, following that pathway literally further and further, one turns out to be in "cul-de-sac", in Hegel's "bad infinity" of new and new, more and more fundamental arithmetical theories, each of which underlies the next one, however postponing the ultimate proof of the mathematical consistent completeness to the time when "Achilles would catch up with the turtle“.

On the contrary, physical and mathematical transcendentalism following and generalizing the success of quantum mechanics to be as complete as consistent replaces that "bad infinity" with idempotency: for example, as reality justifies all the mathematical models or mathematics at all, as mathematics justifies all empirical or experimental facts or reality at all (eventually and as after quantum mechanics, the two sides of idempotency are or can be "complementary"). Then, that idempotency is reconstructed by means of the above analogy: as mathematics justifies all arithmetical models or arithmetic at all, as arithmetic justifies all mathematical theories or mathematics (eventually again "complimentarily").

Still a few simplifications are possible. Mathematics can be represented equivalently.as the composite theory of both arithmetic and set theory in virtue of the Lövenheim - Skolem theorem. Then the idemptency is modified to the following: arithmetic and the system of arithmetic and set theory justifies each other mutually, and possibly, complimentarily:

If mutuality is the case, the idempotency means only that arithmetic implies the equivalence of arithmetic and set theory (after the simplification of the logical expression). This is not sufficient as far as arithmetic can be false as well as set theory, and thus, mathematics. So, the Gödel conclusion seems to be circumvented adding a second arithmetic, in relation to set theory unlike the first one being by itself. However, the success is not sufficient, the ultimate consistent completeness is not reached yet for arithmetic can be still false. The option of mutability for the idempotency has to be rejected therefore giving way to the other one, that of complementarity.

Truly, the option of complementarity wins the ultimate solution since it allows for the fundamental, formal and logical tautology of transcendentalism to be applied. This is due to the consideration of arithmetic, on the one hand, and the system of arithmetic and set theory to constitute

${ }^{20}$ In detail in: Penchev 2020 August 25. 
a "bit of information". Then, the tautology implies for arithmetic to be true as the logical totality. After that, set theory is also true, and mathematics as well. One can apply, fastest, the option of mutuality to demonstrate this as far as the options of both mutuality and complementarity constitutes in turn a bit of information therefore being consistent to the totality, and particularly, to the totality of arithmetic.

A still one simplification is possible if one removes the "brackets" designating the system of arithmetic and set theory and substituting them by others, in which the two twin arithmetics are bracketed, and set theory (mathematics without arithmetic) stays by itself, separately and alone. In other words, the totality of arithmetic can be considered in two different, but equivalent ways in relation to mathematics: (1) arithmetic in relation to the entire mathematics including arithmetic necessarily (recollecting the Lagrangian "reading" of the Schrödinger equation); (2) a generalized arithmetic consist of two twin complementarity twins in turn complementary to mathematics without arithmetic (a "Hamiltonian" allusion).

The consideration until now suffers from the disadvantage that the twin complementary arithmetic is not demonstrated explicitly and constructively yet. Thus, it as well as the generalized arithmetic cannot be utilized as a tool for resolving the most essential and difficult problems of mathematics. That defect is removable as follows. The fact that the second and complementary Peano arithmetic is related to set theory can be interpreted constructively after the following additional postulate about it:

It starts from the least infinite ordinal (usually designated as " $a$ ") just as the standard twin begins by " 1 ". The function successor is defined as " $n-1$ " oppositely to the standard arithmetic: $a, a-$ $1, a-2, \ldots a-n \ldots$ correspond unambiguously to: $1,2,3, \ldots n+1, \ldots$ Then one involves the wellordered series of "natural bijections":

$$
1 \leftrightarrow a, 2 \leftrightarrow a-1,3 \leftrightarrow a-2, \ldots, n \leftrightarrow a-n+1, \ldots
$$

Thus, a new Peano arithmetic is defined obviously isomorphic to each of both complementary twins, and particularly to the standard twin ${ }^{21}$. One can notice that as any bijection as the system of the arithmetic of the bijections and both complementary Peano twin arithmetic constitutes a "bit of information" and therefore allow for Peano arithmetic, on the one hand, and the generalized Peano arithmetic (definiable as that system), on the other hand, to be adopted as the mathematical totality both consistent and complete: to be adopted in virtue of the fundamental, formal and logical tautology of transcendentality in Subsection 6 above.

One can check whether or how that scheme involving the generalized Peano arithmetic is able to circumvent the Gödel (1931) restrictions:

The idea of that "how" can be suggested by the following obvious observation. The Gödel statement claiming for itself to be irresolvable belongs to the class of the "Liar" paradox. However, the latter paradox can be overcome by the Liar himself or herself. She or he knows very well and

\footnotetext{
${ }^{21}$ Utilizing the idea of that new Peano arithmetic one can prove Fermat's last theorem by induction, only arthmetically and shockingly elementarily (Penchev 2020 August 10). Furthermore, one can prove that it is a Gõdel irresolvable statement if it is defined within the system of Peano arithmetic and set theory (for example, as a corollary from the modularity theorem following Wiles's proof), by means of Yablo's paradox (Penchev 2020 August 25). This implies that the proof of modularity theorem involves necessarily inaccessible countable ordinals therefore going out of the standard (ZFC) set theory as far as the arithmetical proof by the new interpretation of Peano arithmetic consisting of those bijections involves countable transfinite ordinals whether explicitly or implicitly, However, they can be removed in the ultimate, elementarily arithmetical proof similarly to "Wittgenstein's ladder" for the isomorphism with the standard Peano arithmetic
} 
unambiguously whether is lying or not. The only problem consists in no way to communicate the internal state being namely a liar: apropos, a property ascribable to any quantum system ${ }^{22}$.

Then, the second complementary Peano arithmetic can be interpreted heuristically ${ }^{23}$ as an arithmetic of the internal states oppositely to the standard twin being that of the external ones. Then the generalized Peano arithmetic refers to both external and internal states therefore embodying particularly the principle of physical and mathematical transcendentalism. What is the case of the Gödel statement?

Its Gödel number is a transfinite enumerable number necessarily as far as it has to contain all natural numbers (Penchev 2010). Thus, it will be a usual, finite number, but in the other twin rather than in the initial, standard Peano arithmetic. This is an idempotency obviously. The justification of each twin consists in the other twin (deducible also still from the logical structure of the Gödel statement to be "out of itself", i.e. "in the other twin"). If one adds the standard Peano arithmetic however interpreted in the bijections between the two twins, the Gödel argument about "either incompletes or inconsistency" will be circumvented just as this was intended: that is, utilizing the generalized Peano arithmetic though explicitly but only as "Wittgenstein's ladder".

(8) Russell's paradox (1902) in Cantor's set theory as an exemplification of the problems about the mathematical totality:

The fundamental, formal and logical tautology implies that paradox.

At first glance, this rejects the tautology as universal and applicable to the totality, for which it is involved properly and thus becoming meaningless. The subsection, however, will advocate the opposite thesis: the tautology resolves the paradox, and even, that solution is equivalently implemented in quantum mechanics, in its mathematical formalism furthermore corroborated very well experimentally.

"Dark matter" and "dark energy" can be interpreted as a direct corollary from the successful solution of Russell's ostentatious antinomy by nature and physics without any contradiction. Thus, those "dark characters" of the discourse appear first explicitly rather than only "mentioned by others". The tautology involving them, therefore implies for the foundation of the Planck constant otherwise usually only postulated an experimental fact, in fact rather shocking, to be justified by it. More precisely, only the necessary existence of the class of constants, to which it belongs, can be forecast rather than its exact magnitude. Nonetheless, that ground needs the direct transformation of (quantum) information into mass and energy both forbidden by "Mach's principle" (Einstein 1918) in general relativity and appearing as dark matter and dark energy on the "cognitive screen" presupposed by Mach's principle: if gravitational field can be caused only by mass and energy according to it, but this is only a convention in fact, any other source (supposedly, such as information whether quantum, or classical) is to be projected on that "screen" as mass and energy, withal just "dark", which means that their origin is hidden, remaining invisible "behind the screen".

That explanation implies still one testable conclusion (though exceptionally shocking): an extremely subtle effect commensurable with the Plank constant should distinguish the same computer

\footnotetext{
${ }^{22}$ One can say loosely that quantum mechanics is created to circumvent the antinomy of the Liar (what any quantum system is by itself) in order to be able to be an objective and experimental science. However, in " 9 " further, that loose, as if only figurative statement will be interpreted absolutely rigorously, formally and mathematically involving the set-theoretical, Dedekind finiteness as the complementary counterpart of the usual, arithmetical finiteness.

${ }^{23}$ And not only heuristically, but the rigorous interpretation is bulky and requires another, postponed in the future paper to be justified in detail.
} 
once in a working state and then, in a non-working state. The working state is featured by a certain flow of information equivalent to an equivalent and exceptionally small, but nonzero change of the quantity of mass and energy in comparison with the non-working state.

In other words, the working computer would produce dark matter and energy, and a relevant experiment would be able to confirm or reject this. The same experiment feasible and conducted on planet Earth would prove the origin of dark matter and dark energy anywhere locally as well as globally in the universe at the same time ${ }^{24}$.

The above synopsis of the subsection will be extended in detail and rigorously:

The starting observation is that Russell's paradox exemplifies the tautology. In fact, Russell and Whitehead devised the plan of "Principlia Mathematica" (known today as "logicism") to justify all the building of mathematics on the base of logic since that of set theory was not reliable enough as the paradox at issue demonstrated. However, its deduction from a rather elementary logical tautology intended here would show that logic is just as vulnerable as set theory and correspondingly, trustless to be the ground of mathematics: that is not the whole truth, though. Indeed, the antinomy follows from the tautology, but the latter verifies the former rather than the former to reject the latter after the relevant "Copernican" change of Gestalt meant by physical and mathematical transcendentalism.

Here is how in detail. One can define a set theoretical operation "doubling" mapping any set to a corresponding pair of sets unambiguously (i.e. as a bijection); the same set not containing itself and the same set containing itself as an additional element. Then, all sets of the former kind constitutes the set of all sets not belonging to itself and necessary for the expression of Russell's aporia (the latter kind is not paradoxical).

However and before that, the so-defined set theoretical "doubling" satisfies the logical expression of a "bit of information", i.e. the "right side" 25 of the tautology, and thus, that "right side" being a necessary condition of the tautology itself implies in turn Russell's irresolvableness: the tautology implies the antinomy. Indeed, the "left side" states for a "bit of information" to be the set of all sets (,) eventually doubled or not by the aforementioned set-theoretical "doubling" (i.e. as a "bit of information' as the entire structure) as a particular case of the totality (defined by the "left side" logically): the totality in terms of set-тхеоретицал totality implies the paradox.

\footnotetext{
${ }^{24}$ Though that conjecture seems to be too radical, it implies an even more radical conclusion though rather speculative. The bodies in a relatively moving reference frame increase their mass and energy to the reference frame of the observer according to special relativity. However, the same enlargement can be due to informational processes at rest to the observer. A principle more general than that of the so-called general relativity and meaning the invariance to any reference frame in a smooth relative movement to the observer. The informational processes (representable equivalently as mechanical motion to discrete reference frames generalizing the concepts of reference in the theory of relativity) are equivalent and indistinguishable from the discrete relative mechanical motion to the observer's reference frame. A visualization: one watches the same motion on the screen of a computer and in reality under the condition that the conjectured mass and energy of the corresponding informational processes for the motion on a certain body to be represented on the screen are fundamentally and exactly equal to them in reality. Obviously, the "screen" and reality would be indistinguishable from each other if that is the case. That coincidence would be implied by physical and mathematical transcendentalism for the physical totality needs the identity of the "screen" and reality. Then, the expansion of the universe can be investigated as more and complicated informational processes more and more remote from the observer because of the exponential growth of the number of independent variables for calculating the state.

${ }^{25}$ The quotation marks mean the conventional, but necessarily disjunctive distinction of the "right side" to the "left side" of the tautology.
} 
However, physical and mathematical transcendentalism changes the Gestalt of the interpretation of the tautology in a "Copernican" way, in fact, implemented and experimentally confirmed very, very well by quantum mechanics. The opposite states are to be considered as "complementary" i.e. in the unified structure of a bit of information, in which they are the two possible alternative choices: " 0 " and " 1 ", for example. Thus and particularly, the set theoretical doubling exemplifying the same type of solution is consistent in virtue of complementarity. Then, the tautology implies Russell's "paradox" not as a real paradox, but as a proposition stating a definitive property of the totality only interpreted in terms of set theory, however not les in those of logic: though propositional logic is equivalent to set theory (at least as Boolean algebras, a more generally: by the principle establishing the bijection of sets and propositions), both are neither contradictory nor inconsistent, neither by each itself, separately nor relatively, to each other after the "Copernican revolution" of physical and mathematical transcendentalism.

One can trace the origin of "dark matter" and "dark energy" still to the Russell's argument (i.e. the antinomy realized a "legal" consistent proposition) even rigorously (i.e. formally and logically). That pathway needs the unification of mathematical transcendentalism as the single physical and mathematical transcendentalism as the next subsection "9" will justify. This is the crucial link for a mathematical and logical statement (as Russell's argument is) to imply a physically testable conclusion (as dark matter and energy are) directly, i.e. abandoning the "crutch of visualization" (in essence, as after Kant) by an ostensibly independent physical theory only externally, loosely, and temporarily juxtaposed to a mathematical model as the common dogma of Cartesian dualism imposes: no, the theory and model are the same once the postulate of the totality has been adopted. So and particularly, the formal and logical pathway from Russell's argument to dark matter and energy needs the "totality", respectively physical and mathematical transcendentalism.

A set regardless of whether it contains itself or not determines a class of equivalence of all of its well-orderings of all of its elements (in advance defining conventionally for all sets whether the set itself to be included in the tuple of its elements or not). That class of equivalence defines just one corresponding ordinal number ${ }^{26}$ and respectively, just one corresponding cardinal number. Then, one considers the set of all components of a certain wave function as just that set, and all well-orderings of them are all possible permutations therefore being all unitary transformation of the wave function at issue conserving the energy of the corresponding quantum state.

That consideration is the boundary of both physical transcendentalism, which unifies them into the single physical and mathematical transcendentalism furthermore. The exact line of both distinction and conjunction is the wave function, which is a "point" of the separable complex Hilbert space (or the unitary class of equivalence, to which that "point" belongs) seen from mathematics once, and a state of a quantum system seen from physics twice. One need add the postulate of the totality (respectively transcendentalism, properly physical and mathematical transcendentalism) to be identified a mathematical object with a physical object as the same by the unifying name of "wave function" (discussed in the next subsection in detail). Otherwise, the "cognitive distance" between a physical object and its mathematical model is postulated to be nonzero in any epistemology of the standard Cartesian dualism such as almost all those of modern science (excluding quantum mechanics).

\footnotetext{
${ }^{26}$ Since a physical interpretation is intended, the considered ordinal numbers can be small enough so that both Cantor - Russel and Neumann definitions of "ordinal number" to be applicable and equivalent to each other.
} 
However, that neo-Pythagorean identification is a necessary condition for the rigorous, formal and mathematical pathway from the totality to dark matter and energy. Fortunately, the postulation of totality interpreted physically and mathematically implies it (in the next subsection) rather than being an additional and independent axiom.

Once having crossed the line of demarcation, one can distinguish the well-orderings of the same wave function from each other physically as well, even two equivalent distinctions simultaneously by the mediation of a qubit (the unit of quantum information) for each well-ordering separately: (1) any qubit can mean as an entangled state as an non-entangled state being isomorphic in both cases; (2) any qubit can mean a state of gravitational field in a certain space-time point (both space-time and energy-momentum vectors). This is true since the bits and qubits share the ability of unifying the synchronic and diachronic aspects of change (or mechanical motion particularly) inherently from the totality via the fundamental, formal and logical tautology. So permuting the components of a certain "atemporal" wave function, one acquires many temporal "hypostases" of it, each of which corresponds to a state of gravitational field in all points of space-time.

There exists a rejecting counterargument, at first glance only, to equate wave function and gravitational field in any way: the unitary permutations conserves energy, the change of the states of gravitational field does not it; furthermore, time is not a variable (in the meaning of not existing a corresponding Hermitian operator) in quantum mechanics unlike time in general relativity.

In fact, those two sides of the counterargument cancel each other mutually. In other words, what quantum mechanics does (i.e. conserves energy, but disorders time) is equivalent to what general relativity does (i.e. disorders energy, but conserves time) speaking in a loose sense. The rigorous meaning of the same equivalence is deduced in Section VII as the conservation of quantum information, after which the "disorders" of time (in quantum mechanics) and energy (in general relativity) can be equated reasonably.

The conservation of quantum information can represent in a better, formally and logically way the distinguishing and unifying boundary of physical, on the one hand, and on the other hand mathematical transcendentalism, which is necessary to be crossed in order to be inferred dark matter and energy "transcendentally". Naturally, it is the middle between the first deduction rather meaning mathematics and the latter deduction rather meaning physics.

The first distinction above, "(1) any qubit can mean as an entangled state as an non-entangled state being isomorphic in both cases", interpreted in terms of gravitational field for its counterpart (2) or for Einstein's "Mach's principle" in general relativity is what generates the "dark field" of matter and energy (thus visible only as "dark") on the reason originating still from Russell's argument. Using the metaphor of disorder, the "disorder of time" in quantum mechanics (which entanglement represents in the final analysis) is the disorder of energy in general relativity (which "dark field" represents in the final analysis) under the condition of quantum-information conservation transforming the metaphor into a logical equivalency.

"Dark field" supposedly equivalent to gravitational field in general relativity is introduced to abbreviate the permanent repetition of "dark matter and dark energy" meaning furthermore that dark matter corresponds to the space-time component of gravitational field, and correspondingly dark energy, to the component of energy-momenta. The Einstein field equation elucidates how they have to be equated in any point of space-time. If the so-called cosmological constant (admissibly even variable) is nonzero, the space-time by itself generates directly gravitational field being non-flat, i.e. in virtue of its curvature. 
Thus, the introduction of the member of the cosmological constant (involved in Einstein 1918 just to satisfy "Mach's principle" also and simultaneously launched then in the same paper) or not demonstrates in fact the "incompleteness of general relativity" (just oppositely to the incompleteness of quantum mechanics only alleged by Einstein and other physicists) in both cases. Einstein himself explained why the zero cosmological constant means the violation of Mach's principle.

However, its introduction also violates it for the space-time itself and by itself (being neither energy nor mass) generates gravitational field. That ostensibly contradictory property of gravitational field is not only consistent, but even very well justified by the entanglement theory of gravity: the space-time curvature in any point is due to the total entanglement in that point.

Particularly, this implies for the cosmological constant to be a variable in general, an energymomentum field corresponding to the dark energy of dark field while the explicit component of spacetime curvature means the counterpart of dark matter in it. Entanglement being instantaneous (Einstein's "spooky action at a distance" therefore expressing his opinion to be absolutely inaccessible) is what "messes up and disjoints" the time of quantum mechanics, a therefore to be conserved energy, which is in turn "messed up and disjointed" in general relativity.

Truly, entanglement can be thought as interacting "parallel universes", each of which featured by its time, but those many times result into the plural time namely "messed up and disjointed" in quantum mechanics. On the contrary, general relativity represents the same figurative interaction of parallel universes keeping the unified time of all of them, but compensatorily needing to "mess up and disjoint" energy from point to point however conserving the classical smoothness for the unified time. Then, the additional "dark field" appears onto the "screen" of the unified time postulated by general relativity as obligatory, but being only a human convention in fact.

Speaking more precisely, any qubit defines a class of equivalence of a large set of entangled states. That qubit by itself corresponds unambiguously to the mass and energy of a certain space-time point. However, as that class of equivalence adds still much more mass and energy as the contribution of "dark field" by the contemporary physics than the visible ones in that point. Meaning that explanation, one can notice that the phenomenon of dark field follows still from the fundamental mathematical concept of "class of equivalence" and implemented initially to be constituted quantum mechanics as both exact and experimental science.

For example, one can define the general notion of a quantity being the difference between a "zero" element of a class of equivalence and all the elements of that class as mapped into any number field (in a meaning algebraic rather than physical) by a function arbitrary in general, but determined equivocally by quantum mechanics and general relativity in the case of dark field.

After that mathematical "quantity of dark contribution" whatever be, but particularly that of dark field, one can reach the pathway, on which Russell's argument implies that set-theoretical and mathematical "dark contribution":

The totality by itself generates change, "philosophical movement" as Hegel's dialects states ontologically, and physical and mathematical dialects postulates as a principle implying experimentally testable conclusions and thus refutable as them. The totality turns out to be a source of change by itself in virtue of the necessity to include its externality within it in order to be still the totality.

The same general observation takes place after the Russell's argument in relation to set theory. The set of all sets, each of which does not belong to itself, is contradictory unlike its counterpart where each of which belongs to itself. However, the latter must be the case that the set of all sets is only mathematical hypostasis of the totality. Then, the obligatory addition of the set itself to itself 
would be to be the relevant expression of dialectics as to set theory. The set added as a whole to itself, on the other hand, represents the necessary class of equivalence resulting in the set-theoretical "dark contribution" and exemplified further as dark field after quantum mechanics and general relativity.

Summarizing the deduction from Russell's argument, one sees that only a specific function interpretable physically determines what exact is "dark field" within of the "class of dark contribution".

(9) The "(in)completeness of quantum mechanics" as the physical totality, but linked to the mathematical totality via the "consistent completeness of mathematics":

One can symbolize the totality unified as physical as mathematical also by rejecting, by Gödel's incompleteness of arithmetic to set theory or by Einstein's one of quantum mechanics to classical mechanics (including special and general relativity) as the same from the new viewpoint meant in the present paper. Both Gödel and Einstein, close friends in Princeton, refugees from Nazi Germany (and maybe for that, convinced opponents of the totality even in science and philosophy) and great, exceptionally famous scientists, can be involved here also as documentary characters of the discourse of the totality and completeness:

The former incompleteness (of Gödel) and the latter incompleteness (of Einstein) can be considered as complementary to each other in the rigorous meaning of Niels Bohr implemented in quantum mechanics. Thus, complementarity implied to itself implies its two aspects of both complementarity and identity complementary to each other and in each other. That applicability of complementarity to itself is a property only of the totality as the fundamental, formal and logical tautology verifies.

If the totality is rejected fundamentally and categorically (as this can be suggested as to the characters of Gödel and Einstein), the two kinds of incompleteness remain absolutely disjoint (and opposite to their togetherness). On the contrary, if one suggests the analogical "fellowship" of both kinds of incompleteness (as the factual one of the two scientists), this implies the totality, on the one hand, and their complementarity rather than disunity, on the other hand.

Of course, to be introduced characters though documentary in a scientific discourse is rather exotic if not even ridiculous, but nonetheless they as personae can designate the abstract conception in a human dimension as in any narrative. The meant idea symbolized by them was introduced already above as Hilbert arithmetic. However, it will be developed essentially now by complementing it with the separable complex Hilbert space of quantum mechanics as its twin counterpart even complementary in the rigorous meaning as well as their identification (being necessary as to the totality) as a nonstandard interpretation of Peano arithmetic ${ }^{27}$.

One need interpret the complimentary Peano arithmetic in terms of the initial Peano arithmetic therefore being transfinite ordinal numbers or "transfinite natural numbers" unlike the finite natural numbers of the former. Then, the Dedekind, set-theoretical finiteness is to be involved to distinguish the transfinite ordinal numbers from each other in relation the finite ones of the complimentary counterpart as follows:

If one means the mapping of any set, to which a transfinite ordinal (natural) number corresponds one-to-one, into the $\operatorname{set}^{28}$ of all sets, to which a certain finite natural number coincides with (corresponds unambiguously to) its cardinal number, under the condition for the image, an element

\footnotetext{
${ }^{27}$ The conception in detail is available in a previous paper (Penchev 2020 August 25), and will be only sketched here as the "forest" without the "trees".

${ }^{28}$ That set is different from the set of all natural numbers, one can notice, since the former is finite, and the latter is actually infinite.
} 
of the latter, to be chosen only by the means of the axiom of choice, i.e. fundamentally randomly excluding any hidden variables partly or thoroughly predetermining the choice. A corresponding probability density distribution will appear after any mapping of an infinite set into a finite set. That mapping satisfies the condition of Dedekind finiteness since it cannot be any bijection, even any function at all, being fundamentally ambiguous. So, the corresponding probability density distribution can be accepted definitively as the corresponding finite expression of the transfinite natural number.

The most important proof from a properly mathematical viewpoint consists in verifying the bijections of the set of all transfinite natural numbers and the set of all probability distributions of finite natural numbers, built as above and then, to all wave functions as the corresponding characteristic functions of those probabilistic density distributions. However, it is bulky rather technical than conceptual, and its publication is postponed to a future paper: thus, omitted here.

Then, the result is a bijection of the separable complex Hilbert space into the transfinite natural numbers since they would be seen from the usual Peano arithmetic as probability density distributions of finite natural numbers. Then, Hilbert arithmetic consisting of two dual Peano arithmetics is doubled itself as still two identical separable complex Hilbert spaces, identical in the meaning of anti-isometry, and thus, interpretable as dual.

The aspect of duality (i.e. complementarity in a narrow sense) of Hilbert arithmetic and the separable complex Hilbert space can be granted as proved.

However, the aspect of identity of them is even more surprising if not shocking. Its proof relies on involving a nonstandard interpretation of Peano arithmetic. Here, the sense and meaning of "nonstandard" is opposite to the usual one: the nonstandard interpretation means an arithmetic model of any mathematical structure in virtue of the Löwenhem - Skolem theorem. On the contrary, a model of Peano arithmetic into set theory and propositional logic is meant now and explicitly by a relevant interpretation of the Peano axioms:

The problem is whether the well-ordering is a necessary condition of the Peano axioms or not. Only the axiom defining the function successor as " $n+1$ " implies it and can be considered as a necessary condition. However, the problem is not resolved, in fact, if one means arithmetic as the ultimate base of mathematics since the operation "+" would be mentioned for a first time just in that axiom and definable only contextual in what arithmetic means. Thus, though "+" is interpreted standardly as the operation "addition" in the traditional arithmetic or specified moreover in algebra following from arithmetic formally and logically, any other interpretation of "+" would be possible not less as long as it would not contradict the Peano axioms. For example "+" might be interpreted as the "conjunction" in any Boolean algebra such as that isomorphic to propositional logic or the settheoretical algebra, and then the function successor " $S(n)=n \vee 1=n \cap 1=n$ " implies the consistent interpretation of the function successor as " $S(n)=n$ " and the following bijection between the standard and new interpretations: $1 \leftrightarrow 1,2 \leftrightarrow 1=1,3 \leftrightarrow 1=1=1, \ldots$

Furthermore, the interpretation can satisfy the complimentary arithmetic where, speaking loosely, it starts from the other end or extreme of the infinite chain of equalities: $a \leftrightarrow a, a-1 \leftrightarrow a=a, a-$ $2=1+1+1 \ldots$

Both bijections are isomorphic to each other obviously, add the isomorphism implies $a=1$. On the one hand, this means that the nonstandard Peano arithmetic is cyclic and closed unlike the open standard Peano arithmetic. In other words, arithmetic is "clopen" in virtue of the Peano axioms only, and this is absolutely consistent to usual utilization of "clopen" in topology, valid to discrete topology such as that of Peano arithmetic. 
On the other hand, the conclusion " $a=1$ " defines an infinite sequence of equalities, which can serves as a purely arithmetical definition of "infinite set", namely that being able to be mapped oneto-one to the above series of bijections. The option for infinity to be defined arithmetically demonstrates particularly that the nonstandard interpretation (unlike the standard one) of Peano arithmetic is consistent to set theory which can be verified directly discussing either validity or invalidity of Gödel's incompleteness to it.

How is the Gödel proposition stating to be valid via the necessary mediation of its Gödel number modified in relation to the nonstandard Peano arithmetic?

The Gödel numbers of all possible propositions are different, but equal to a unit after the interpretation of the function successor as equality. The expression "different, but equal to a unit" is not contradictory in any Boolean algebra presupposed implicitly to the nonstandard interpretation of Peano arithmetic. Then, if there exists at least one resolvable proposition after the Gödel numeration the Gödel proposition is resolvable also as a necessary corollary. Speaking loosely, if there exists truth at all, the Gödel proposition is resolvable after the nonstandard interpretation.

For more persuasiveness, one can track why the mechanism of the Gödel proposition to generate a fundamental trouble stops working after the new interpretation. The mechanism outlined in the standard interpretation: one the one hand, the Gödel proposition possesses a finite natural number as its Gödel number, on the other hand, it implies a transfinite natural number as its counterpart. If both counterparts are identified, a contradiction appears since a finite natural number is equated to a transfinite natural number. If both counterparts are distinguished, arithmetic is incomplete in relation to set theory since the transfinite natural numbers cannot be incorporated in it consistently.

The mechanism modified after the nonstandard interpretation: the finite and transfinite natural numbers can be as distinguished as equated to each other as two disjunctive interpretations of the same: Indeed, the finite natural numbers belong to $1 \leftrightarrow 1,2 \leftrightarrow 1=1,3 \leftrightarrow 1=1=1, \ldots \quad$, and the transfinite natural numbers belong to $a \leftrightarrow a, a-1 \leftrightarrow a=a, a-2=1+1+1 \ldots$. However, both are furthermore isomorphic to each other and the same (in that meaning of isomorphism). The above dichotomy has become impossible: nor the contradiction appears since the finite and the transfinite numbers are equated in definition, nor arithmetic is incomplete because the transfinite numbers are incorporated consistently.

The solution can visualized in terms of the antinomy of the Liar still more that the Gödel paper itself refers to the Liar as a more loose formulation of what is defined rigorously, formally and logical as the special proposition called by his name later.

It admits very simple, but rather instructive solution if one changes the Gestalt. In fact, the Liar himself or herself knows very well and equivocally, that she or he is lying or not. However, this knowledge is not communicable as far as the Liar is a liar.

Rather intriguing: any quantum system is fundamentally incapable to communicate its state and thus, considerable as isomorphic logically to the Liar. Then, quantum mechanics can be realized also as a way to be built an exact, experimental and objective science about ... the Liar's statements in the final analysis: any measurement is a Liar statement and thus arbitrary. However quantum mechanics studies only the "profile of the Liar" since any liar (quantum system) in the class of the Liar can be distinguished from any other liar by its "profile" (wave function).

Well, the identification of Hilbert arithmetic and the separable complex Hilbert space implies the interpretation of that profile (wave function) with a certain transfinite natural number and then, the Gödel proposition number (i.e. the Gödel number of the Gödel proposition) is a certain profile among 
those. Though it is irresolvable, its "profile" is resolvable: it either belongs to the liar at issue (among the class of the Liar) or not.

The metaphor of the "profile" of the Liar (apropos, interpreted absolutely rigorously und unambiguously here) conserves the external viewpoint to the Liar studied by scientists, admissibly in the area of quantum mechanics. This corresponds to the viewpoint from an arithmetical number only from the other reference frame of the complimentary twin of Peano arithmetic. However, it is resolvable definitively in its own reference frame. As to the Liar, this needs the change of Gestalt, which means the Liar proposition to be seen in the Liar's consciousness as for as the Liar cannot help but know whether he or she is lying or not, but is not able to communicate that knowledge definitively.

Just the identification of the profile of the Liar with the Liar's internal knowledge as the identification of the objective psychological cognition with the subjective introspective knowledge is very, very instructive and probably very fruitful to the mathematization of psychology, the philosophical doctrine of dualism or both in Husserl's phenomenology

Passing to a more abstract viewpoint, the essence of the solution is the following. Set theory is clopen. The standard interpretation of arithmetic is only open. The argument of Gödel's incompleteness claims the property of being also clopen is contradictory to Peano arithmetic and thus it is necessarily open but not closed. The nonstandard interpretation of Peano arithmetic demonstrates that this not true and it admits as (two) open interpretation(s) (eventually complementary to each other) as a clopen interpretation. Thus, the Gödel issue is removed: no obstacle for arithmetic to be consistent to set theory and for both to be the reliable foundations of mathematics.

The properly philosophical reflection from the viewpoint of physical and mathematical transcendentalism is the following. The consistent completeness of mathematics exemplifies the totality in its terms. The totality would be also clopen if one admits a mathematical concept to the most fundamental philosophical one, and transcendentalism including physical and mathematical transcendentalism for the relevant translation being much more than a loose metaphor. The aspect of the externality of the totality means for it to be open, and the necessary transcendental mapping of the transcendent externality into the immanent internality implies for it to be closed. The consistent reconciliation of both aspects of the totality (furthermore definitive for it) implies for it to be clopen as well as transcendentalism, and then physical and mathematical transcendentalism.

Hilbert arithmetic includes the two complimentary standard interpretations of Peano arithmetic together with its nonstandard interpretation, therefore consisting of triples of elements of the same name, each of which in one from the three interpretations of Peano arithmetic. That triple of the elements of the same name is isomorphic to a bit of information. Yes, the element belonging to the nonstandard interpretation corresponds to the state before choice (meaning a bit of information), e.g. the empty tape cell of Turing machine, that of the one twin, the one alternative of the choice, e.g. "1" recorded in the tape cell of Turing machine, that of the other twin, the other alternative of the choice, e.g. "0" recorded in the same cell.

Furthermore, any bit represents a Boolean variable. Thus, the Boolean algebra on Hilbert arithmetic can be defined quite naturally on the class of the auto-morphisms of $2^{n}$ ordered bits, or particularly their mappings into a single bit as the basic Boolean operations. For example:

$$
\begin{aligned}
& \wedge \stackrel{\text { def }}{=}[(0,0) \rightarrow 0],[(0,1) \rightarrow 0],[(1,0) \rightarrow 0],[(1,1) \rightarrow 1] ; \\
& " \vee " \stackrel{\text { def }}{=}[(0,0) \rightarrow 0],[(0,1) \rightarrow 1],[(1,0) \rightarrow 1],[(1,1) \rightarrow 1] ; \\
& " \neg " \stackrel{\text { def }}{=}[0 \rightarrow 1],[1 \rightarrow 0]
\end{aligned}
$$


(10) The relation or union of mathematics and physics as the physical and mathematical totality:

The main conjecture, which follows from the discussion until now in relation to the mathematical and physical totality (and originating from the view of the totality in the philosophy) can be synthesized in a theorem, the rigorous proof of which will be postponed for a future paper though the sketch is outlined already here:

\section{Conjecture:}

Statement 1. Both set theory and propositional logic possess the same consistent model in Hilbert arithmetic.

Statement 2. Hilbert arithmetic is isomorphic to the separable complex Hilbert space (utilized in quantum mechanics).

Statement 3. Hilbert arithmetic contains (implies) an internal proof of consistent completeness.

Granting the conjecture to have been proved ultimately, a few conclusions properly philosophical or scientific and philosophical will be enumerated and outlined here:

The consistent completeness of mathematics by an internal proof can be reached only in a transcendental pathway including the non-mathematical externality, i.e. the world by itself into and only within mathematics (thus recollecting Pythagoreanism). The statement 2 is what supplies that necessary condition by the "Januslike" or twofoldly interpretable "wave function": the one "face" is purely arithmetical and also Januslike or twofoldly, in turn, as both "transfinite natural numbers" (as the finite natural numbers are seen from the "reference frame" of the other, complementary twin) and finite natural numberc (as the finite natural numbers are seen from the own "reference frame" of each twin); and the other "face" is a state of a quantum system, what quantum mechanics claims to be for any physical entity: there exists no physical entity without being a quantum system: an absolute rule thoroughly.

However, the last statement is not sufficient yet for all the world to be includible in mathematics. What is also necessary is to be considered the states of a quantum system as a generalization of the same system, after which "quantum system" (i.e. by itself) would be a special, "zero" state among all states of the same system. That additional necessary condition is the fundamental law of quantuminformation conservation, from which follows particularly that matter and energy featuring anything physical in the so-called visible universe (unlike the "dark universe" attributable with dark field) are only that special, "zero" state of quantum information definable by time physically or by wellordering mathematically.

The contemporary observations underestimating presumably the contribution of the dark universe, nonetheless, determines the participation of the visible universe as 4-5\% approximately.

Speaking metaphorically and even poetically, humankind reaches the ocean shore of the dark universe and understands that lives in a small island of the visible universe seeming to be all the universe until now and struck by vastness and immensity: what was all before has become an island now and one can suggest that new and new horizons will reveal one by one further, too.

The generalization conserving quantum information (and only particularly and under additional conditions, matter and energy) can explain dark field and hence, the dark universe, by that part of quantum information being not well-ordered mathematically and out of time (but not out of space) physically, and representable of the screen of time (to the kind of which the cognitive screen of general relativity belongs as well) as matter and energy inherently "dark" being behind that "screen" and only projectable on it.

A relatively old, but rather technical concept, that of information acquires a fundamental status both scientific and philosophical as the initial element able to generate all the visible universe as an 
"island" or "screen" in it: all is quantum information and it can be thought philosophically to be the same as the totality or self-generating itself by itself in virtue of transcendentalism and dialectics, both, philosophical and mathematical and physical.

Information is defined by any triple of the same name (or of the same number, said more precisely), in which any element belongs to just one from the nonstandard interpretation and both complimentary interpretation of Peano arithmetic. Particularly, ordered tuples of bits of information defines Boolean algebra shared and therefore unifying both propositional logic and set theory suggesting an isomorphism of parts or aspects of them at least or even an absolute isomorphism under certain conditions to be investigated.

Introducing the unit of quantum information, a qubit as usual (as quantum mechanics does it) and proving that quantum information involved so is equivalent to the information of infinite series or sets (Penchev 2020 July 10), the separable complex Hilbert space being the physical "twin" of Hilbert arithmetic, which is the properly mathematical "twin" among both, shares the same "genetic" foundation, the same "DNA" of information (what the DNA itself, without quotation marks represents in fact, by the way) involving a cyclic structure, or the physical and mathematical dialectics of the "whole and parts" therefore involving explicitly how any part is the whole at the same time; and here is how:

Hilbert arithmetic can be inferred form the separable complex Hilbert space represented equivalently in advance as the qubit Hilbert space (or qubit space further for briefness), after the class of equivalence of all values of (i.e. recordable in) an empty qubit has been notated as a unit of Hilbert arithmetic, i.e. a single bit of classical information, the counterpart of " 1 " in Peano arithmetic ${ }^{29}$. Then, the "global space" of qubit space being an infinite set or series of classical bits is represented in turn in any single qubit internally, thoroughly within it. Any possible "record" in a single qubit is a state of the global qubit space being an infinite series or set of bits of classical information. That representation is possible only because of the definitive property of "infinite set or series" to admit bijections to true parts (accordingly, true subseries or true subsets).

Being classes of equivalence, any unit of the global space allows for a redefinition (or more precisely, additional definition) by any value "recorded" in a single qubit ("empty" before that). That redefinition or additional definition can be interpreted as the complimentary viewpoint to the same unit however from the "reference frame" of the other twin as the complimentary qubit of the dual qubit space as a state of entanglement.

The three interpretations are also isomorphic mathematically. In other words, any qubit being the same by itself can be seen from three different (but human) viewpoints as if three ostensibly different things: (1) as a transfinite natural number in Hilbert arithmetic; (2) as the dual counterpart in qubit space; (3) as an (elementary) state of entanglement in the physical theory of quantum information. Just " 3 " implies to be interpreted back, in the global space as a "curved sphere" (i.e. the state of spacetime in general relativity by pseudo-Riemannian space in a single moment of time) seeming as dark field on that kind of cognitive screen and therefore not coinciding with the reality by itself: dark field represents (i.e. projects) the difference between the reality by "screen" and by itself in terms of the "screen".

However, the equivalence and isomorphism of the three, "1", " 2 ", and " 3 " implies for dark field to be trackable to both hypostases of the physical theory of quantum information (i.e. the

\footnotetext{
${ }^{29}$ That counterpart is bijective, but not equivalent, which, in particular, is able also to explain the consistent completeness of Hilbert arithmetic in comparison with the Gödel dilemma: either incompleteness or inconsistency of Peano arithmetic to set theory.
} 
mathematical counterparts of the separable complex Hilbert space borrowed by physics from geometry) and Hilbert arithmetic.

The unity of the physical theory of quantum information and the separable complex Hilbert space and Hilbert arithmetic is underlain in turn by the totality of physical and mathematical transcendentalism (as a falsifiable scientific conception) or by the totality of philosophical transcendentalism (as a metaphysical conception in Popper's sense ${ }^{30}$ ).

(11) The relations and functional dependences of the mechanical description of a system to its holistic description in phenomenological thermodynamics by different (e.g. Boltzmann's, Gibbs's Einstein's) versions of statistical thermodynamics:

The concept of physical and mathematical transcendentalism has been available in classical physics implicitly anyway. The equivalence of the Lagrangian and Hamiltonian formulations (applicable to quantum mechanics furthermore) was discussed already,

However, there exists still one example, thermodynamics, once phenomenological, but twice statistical, or the statistical thermodynamics itself, once Boltzmann's, twice Gibbs's, and even thrice, Einstein's. One cam notice that relation of statistical and phenomenological thermodynamics embodies the main idea of physical transcendentalism in the following form:

A special kind of physical quantities, namely thermodynamic and phenomenological, are attributed to a macroscopic whole, such as pressure, volume, temperature, entropy, and others, being at the same time only emergent, referring to the whole at issue, but not at all to the elements whatever they have been postulated by each of the three modifications (though partly equivalent to each other) of statistical thermodynamics.

On the other hand, the mechanical quantities featuring the elements of any kind of statistical thermodynamics and united in a huge ensemble generate probably (eventually, density) distributions and a few options of average values resulting in the macroscopic phenomenological thermodynamic quantities in a different way specific for each modification. Energy and time are the universal quantities valid to both mechanical elements and thermodynamic whole.

From the viewpoint of physical and mathematical transcendentalism, the approach of statistical thermodynamics can be described so. The phenomenological thermodynamic quantities mean classes of equivalence of all probability (density) distributions and average quantities which result in the same values of a phenomenological thermodynamic quantity as a single one as a set of them (a macroscopic thermodynamic state). Statistical thermodynamic investigates those classes of equivalence meaning corresponding and different elements in each modification.

Phenomenological thermodynamics describes the system outside, to an external observer, and the Boltzmann statistic thermodynamics, inside, to a hypothetical internal observer. The elements of the Gibbs counterpart, however, are states of the system described usually in phase space doubling the usual Euclidean space of empirical experience and experiments. The Einstein modification adds external (i.e. macroscopic) interaction to the system, or generalizes Gibbs's where those external interactions are granted as zero. Thus, both Gibbs and Einstein versions substitute the hypothetical internal observer of the Boltzmann one by an equivalent external observer distinguishable from that of the phenomenological thermodynamics by registering the generalized mechanical quantities of the

\footnotetext{
${ }^{30}$ The reason for metaphysical conceptions in history of human cognition is to be kept regardless of being confirmed or refused in a certain age for future epochs when they can be resurrected whether modified or not: thus, physical and mathematical transcendentalism refers in the present age in a falsifiable way to the "eternal" philosophical transcendentalism being "metaphysical" as unfalsifiable. The latter is stored for future generations whatever its estimation of the present generation be.
} 
system and describing them in phase space rather than the phenomenological thermodynamic quantities registered by the observer of phenomenological thermodynamic. The classes of equivalence meaning by the latter consists of the elements of states of phase space, i.e. the doubled empirical space.

The Einstein approach investigates, but only in the final analysis, the relation of the Gibbs statistical observer of elements of the class of equivalence, on the one hand, and the phenomenological observer of the whole classes (of equivalence) themselves, and particularly, the condition under which they can be identified as the same ${ }^{31}$. Then, external interactions generate arbitrary changes in the state of the system splitting those two observers to each other in transients, but tending to an identification again establishing an equilibrium.

The approach of Einstein can be considered furthermore as a key of how his general relativity to be considered as a thermodynamic theory referring to the space-time points themselves directly rather than to any system clearly distinguished in a certain area of space-time, which it occupies.

Indeed, gravitational field can be considered in a transition to infinity of a series of Boltzmann descriptions where the elements are smaller and smaller, tending to points, weather material (with a finite nonzero mass at rest) or energetic (with zero mass at rest). Then, the equating of Boltzmann and Gibbs descriptions of gravitational field, all mechanical variables are "cancelled mutually", speaking loosely, and a probabilistic density distribution, that of space-time points, will be equated to another, that of the imaginary states of the universe outside, so to speak, from "God's viewpoint". This equation is only informational: it expresses the mutual entropy "inside and outside" of the universe. The course of thought is inverse to that of the Boltzmann thermodynamics: the real observer is inside, and the conjectured one is outside, external, as it were "God" in the present consideration.

Then, the Boltzmann - Gibbs interpretation of gravitational field can be generalized further, to the Boltzmann - Einstein interpretation of it, i.e. adding an external action on the universe outside, as if an "action of God". It will be leap-like as any quantum leap from a purely information viewpoint as in the above equation of Boltzmann and Gibbs interpretations and therefore able to equate the universe to the scale of a quantum in this relation.

The same discrete change, however, will be represent in the smooth transients of gravitational field "within the universe" and only observable experimentally by humankind (unlike "God's impetus outside"). One can admit and eventually prove that the Einstein field equation describes just the transient, i.e. the smooth representation of the jump-like change as it were outside. The constant of light speed in a vacuum is to feature the "elasticity of space-time" as a medium, determining the propagation speed of any, as if "external impetus" smoothly and gradually ${ }^{32}$.

31 This corresponds to Neumann's definition of a class of equivalence by the identification with a single element of it as to ordinal numbers in set theory (Neumann 1923).

${ }^{32}$ That representation recollects the "ether theory of light propagation", which is common to be granted as rejected by special relativity. The reason is that it implies Galilean rather than Lorentz transformations, therefore contradicting the postulate for light speed in a vacuum to be a constant. However, if one changes the "immanent" viewpoint inside, shared by special relativity and ether theory to the "transcendent" one, outside, the consistency of the concept of ether and the light speed postulate seems to be almost obvious. The ether theory seen inside suggests that any movement being inside inherently should sum with that of the ether itself. However, if the movement of the ether originates, speaking loosely, from the externality of the universe, it would not be to be summed with any movement within it. On the contrary, the movement caused externally should be the maximal bound of any internal movement therefore elucidating the reason for the light velocity postulate usually involved only ad hoc for the equivocal experiments, as Einstein (1905) did. Another paper (1920) of his advocates the refused ether by the new and wider viewpoint of general relativity. Indeed, the 
One can conject naturally that the purely informational interpretation of gravitational field as above (by the way, in the same direction as Erik Verlinde (2011)'s theory of gravitation) should be equivalent to the quantum-information of general relativity in the present paper and implying entanglement as the source of dark field. If that is the case, a corollary from it would be as the explanation of dark field in the framework of that, thermodynamic interpretation of general relativity, and presumably, by Verlinde's theory as well.

\section{THE OTHER, PHYSICAL POLE OF DARK MATTER \& DARK ENERGY}

Unlike, the former pole of transcendentalism, or even in the form of physical and mathematical transcendentalism implicitly available a long time ago in human cognition, what is known and well established about dark field is insignificantly small and can be summarized in a few statements:

1. Both dark matter and dark energy are proved experimentally very well.

2. They overcome crucially in the universe being approximately $95-96 \%$ of the total mass and energy of the universe.

3. They can be noticed only after astronomic observations of mega-objects such as galaxies whether ours, the Milky Way or sectors of the universe. (They cannot be registered in any macroscopic or microscopic phenomena after the contemporary exactness of measurement.)

4. They are only and purely gravitational phenomena.

5. They need the model of general relativity as a necessary condition to be articulated as absolutely certain values. So, one can admit that they appear only on the "cognitive screen" of general relativity being a testimony of its defect or incompleteness eventually.

6. No internal structure (besides the opposition of dark matter and dark energy) can be distinguished: they are established only by their total influence on the visible and structurable part of the universe.

7. Dark matter and dark energy differ from each other by the different kind of astronomic observations to be verified: dark matter imitates the gravitational effect of a huge amount of hidden (but visible in general unlike the original counterpart of dark matter) mass at rest holding as a whole a very fast rotating mega-object such as our Milky Way; dark energy is ascertained only by the accelerating expansion of segments of the universe unexceptionally. The latter resembles the effect of the constant inflow of energy anywhere in the visible universe and being due to the energy of hidden sources, however visible in general unlike the counterpart of dark energy.

8. If dark matter and dark energy be commensurable in any, but the same units, the dark matter is approximately $40 \%$ to the dark energy, or approximately $28 \%$ of their total amount, or approximately $27 \%$ of total amount including the matter and energy of the visible universe.

9. Dark matter and dark energy are clearly distinguished from each other also on the cognitive screen of general relativity, i.e. in the Einstein field equation as different members of it: dark matter corresponds unambiguously to the space-time tensor, and dark energy, to the energy-momentum tensor therefore both together constituting an equivalent gravitational "dark field".

10. One can grant even that dark field is meant explicitly, though only totally, in the Einstein field equation by the member containing the "cosmological constant", i.e. as the integral of dark energy to all the space-time being a shared effect of dark and visible mass.

All the rest about dark matter and dark energy are only ideas, suggestions, conjectures, which would be to be consistent to the above facts. Those of the present paper are among them.

present investigation demonstrates that general relativity involves implicitly an observer external to the universe, and to whom the conception of ether is consistent to the postulate of light speed in a vacuum. 


\section{THE INTERMEDIATE STAGE OF QUANTUM INFORMATION CONSERVATION}

The most essential physical hypothesis in the present paper is the suggestion of the conservation of quantum information therefore generalizing further the conservation of mass and energy valid to the visible universe ${ }^{33}$.

Energy, and thus mass (featured as possessing a nonzero mass at rest), imply time, i.e. the physical quantity of the mathematical well-ordering, e.g. as the corresponding Noether counterpart of energy. Quantum mechanics involves a very essential generalization of time, where it is standardly definable directly only to the apparatus, but indirectly, by means of energy conservation and unitarity as to the investigated quantum system.

That direction of physics generalized in relation to time defined weaker and weaker continues after the phenomena of entanglement and the conception of quantum information where a few independent of each other "times", each for each entangled subsystem, and separately, for the system as a whole therefore excluding any unified time otherwise than by convention (what that of the system as a whole to be granted is as usual).

Moreover, even still one and more radical step to be overcome the framework of time is possible: physical objects out of time and thus not possessing properly energy to be postulated (though an equivalent unambiguous mass and energy as those of dark field to be introducible as observable, but only on the cognitive "screen of time").

Those objects share the same most fundamental substance of quantum information. If one whether likens or interprets the universe as a single immense quantum computer, those entities consisting properly only of quantum information would be to be structured as different "calculations", parallel in general, of that quantum computer, and only a small part resulting in the visible physical objects on the temporal "screen" granted by the contemporary physics as all the universe, due to ignorance faintly elucidated only by the discovery of dark field ant its crucial dominance in the universe (being equivalent to the fact for the universe to be the physical totality).

Thus, those "dark objects" behind the "temporal screen" of our experience and experiments are more relevant to be structured as computational processes therefore each of them sharing the wholeness of a single algorithm and only sometimes resulting into the visible physical objects on the "screen of time".

If one involves relevant metaphors and visualizations in Einstein's manner, one can figure us ourselves, all humankind as two-dimensional characters on the screen of an imaginary huge computer. The entire temporal history still even from the Big Bang is not more than all the happened only on the screen, also only and accidentally referable to real calculations in depth. Naturally, we, as the beings on that screen, have granted quite uncritically and even without being able to reflect our

\footnotetext{
${ }^{33}$ One should mean the following ambiguity of the use of the term of "visible universe". Once it refers to the part of the universe visible from our planet at the present moment. Twice, it is related to the part of the universe which our cognition until now is able to distinguish and structure in opposition to dark matter and dark energy. After a relevant philosophical reflection, the two uses are to be separated as belonging to the physical "metaphysics" (ontology) or to its "epistemology" accordingly. Transcendentalism as well as its scientific counterpart, physical and mathematical transcendentalism suggest for ontology and epistemology (respectively, metaphysics in a narrow sense and physics) to be unified in the totality once it has been postulated. This implies the both uses to be identified in turn, however this is not so simple and immediate, but needs another study about the conditions and conclusions if both meanings are identified as the same, being a form or conclusion from the totality since it would call for revolutionary changes in our worldview (similar to those about the "Big Bang" discussed here as well), and thus, would need justification in detail.
} 
actual position in a rather consistent worldview, but explicitly consistent only until the puzzling discovery of dark field.

Thus, what is forthcoming for those screen humans is a unique and unprecedented scientific revolution: the available and common worldview is fundamentally wrong for the happened on the screen has been granted to be the ultimate reality by itself therefore veiling inaccessibly the hidden, but crucial dimension of calculations, of creating the "realty" visible on the screen.

The incredible scale of that scientific revolution can be exemplified by a few specific details:

The interstellar travels as were figured until now, i.e. without leaving the screen ever, are meaningless and even presumably impossible physically. Particularly, this explains the so-called Fermi paradox ${ }^{34}$.

The CERN more and more "super" colliders are rather the meaningless, reckless spending of billions and trillions of money, for the real creation of the universe including the visible one on the screen has not occurred and cannot in principle occur on the screen itself. The Big Bang is a myth due to our ignorance.

Many phenomena seeming mystic and antiscientific will be explained scientifically and clearly distinguished from their charlatan twins thriving on human ignorance as usual.

\section{THE FIRST DEDUCTUION: FROM THE TOTALITY VIA TRANSCENDENTAL INVARIANCE TO QUANTUM INFORMATION CONSERVATION}

In fact, that first deduction was explicated in detail already, and now, what remains is to be systemized and summarized concisely. It is situated within mathematics, but borders philosophy from

\footnotetext{
34 "The remark came while Fermi was discussing with his mealtime mates the possibility that many sophisticated societies populate the Galaxy. They thought it reasonable to assume that we have a lot of cosmic company. But somewhere between one sentence and the next, Fermi's supple brain realized that if this was true, it implied something profound. If there are really a lot of alien societies, then some of them might have spread out. Fermi realized that any civilization with a modest amount of rocket technology and an immodest amount of imperial incentive could rapidly colonize the entire Galaxy. Within ten million years, every star system could be brought under the wing of empire. Ten million years may sound long, but in fact it's quite short compared with the age of the Galaxy, which is roughly ten thousand million years. Colonization of the Milky Way should be a quick exercisable. So what Fermi immediately realized was that the aliens have had more than enough time to pepper the Galaxy with their presence. But looking around, he didn't see any clear indication that they're out and about. This prompted Fermi to ask what was (to him) an obvious question: "where is everybody?" (Shostak 2001 October 25). "As the story goes, Italian physicist Enrico Fermi, most famous for creating the first nuclear reactor, came up with the theory with a casual lunchtime remark in 1950. The implications, however, have had extraterrestrial researchers scratching their heads in the decades since. "Fermi realized that any civilization with a modest amount of rocket technology and an immodest amount of imperial incentive could rapidly colonize the entire galaxy," the Search For Extraterrestrial Intelligence (SETI) Institute in Mountain View, California, said on its website. "Within ten million years, every star system could be brought under the wing of empire. Ten million years may sound long, but in fact it's quite short compared with the age of the galaxy, which is roughly ten thousand million years. Colonization of the Milky Way should be a quick exercise." Fermi reportedly made the initial remark, but he died in 1954. Publication fell to other people, such as Michael Hart, who wrote an article titled "An Explanation for the Absence of Extraterrestrials on Earth" in the Royal Astronomical Society (RAS) Quarterly Journal in 1975. (Some say this is the first such paper to explore the Fermi paradox, although this claim is a bit hard to prove.)" (Howell 2018 April 17). The name of Fermi is not mentioned in any reference as well as in the entire text of the cited paper (Hart 1975) where (presumably independently) the problem known as the "Fermi paradox" is formulated in a scientific article published in a journal for the first time. The paper "Anthropology and the search for extraterrestrial intelligence" (Dick 2006) considers the cultural and scientific background of the paradox (citing it namely as "Fermi paradox") and refers to many original scientific articles.
} 
the one "side" (in the concept of the totality), but physics from the other "side" (in the conservation of quantum information as a most fundamental natural law). Its essence is just that transfer from nonfalsifiable philosophical totality to an experimentally confirmable and thus as verifiable as falsifiable universal statement of the law. The transfer itself is only mathematical and thoroughly according to what is truth in mathematics: logical consistence of the deduction to the conclusion if a few postulated premises are granted.

Two of those postulates rather philosophical and methodological than mathematical are located at the philosophical boundary of mathematics, on the one hand, and at its physical boundary, on the other hand:

The former states that the totality can be identified with the mathematical and physical totality accordingly as consistent completeness and the universe, and the non-falsifiable philosophical transcendentalism, with the physical and mathematical transcendentalism as falsifiable as verifiable.

The later postulates that any element of the mathematical separable complex Hilbert space (i.e. a "wave function" in a purely mathematical meaning) can be identified unambiguously with a certain quantum state (i.e. a "wave function" in a properly physical meaning).

Those two postulates together guarantee that a mathematical statement proved as a theorem can be interpreted as justifying the transfer from the philosophical totality to that generalized physical law. In fact, both postulates contradict and even scandalize the common prejudice prohibiting the direct transfer especially between philosophy and mathematics (the cross of the boundary between mathematics and physics is rather met in theoretical physics nowadays). However, this is not more than a prejudice otherwise violated by "fathers" of the modern mathematics, such as Descartes or Leibniz.

Anyway, they are only postulates, which can be as accepted (in the present paper) as rejected.

The physical and mathematical totality is defined as what contains its externality within itself necessary therefore generating a fundamental doubling isomorphic to a bit of information. Thus, information is the quantity and concept able to mediate between physical and mathematical transcendentalism and the foundations of mathematics by (Peano) arithmetic and (ZFC) set theory. This is a very essential change in the status of "information": from a rather technical notion (e.g. as Shannon introduced it) or mathematical (Kolmogorov) to a fundamental philosophical concept, even category.

The physical and mathematical totality implies (besides doubling) furthermore (and independently) cyclicity. The last element of any well-ordering of the totality (i.e. the totality itself) and the first element (existing necessarily according to the definition of well-ordering) can be identified with each other therefore cancelling the well-ordering and any well-ordering at all. And vice versa: if any cyclicity is granted, its "cutting" defines the totality as the last element of wellordering and the opposite pole of the least element due to the same well-ordering.

One can find both properties of the mathematical totality in the separable complex Hilbert space or represented equivalently in the qubit Hilbert space. Indeed, its unites bits of dual qubits of the same ordinal number, and any qubit being the information of a certain infinite set or series can be considered as a state of the whole qubit Hilbert therefore embodying the definitive property of cyclicty.

The qubit Hilbert space can be proved furthermore as the dual counterpart of Hilbert arithmetic in turn defined on the base of two complimentary copies of Peano arithmetic interpreted standardly (i.e. as two opposite well-orderings) and still one copy of Peano arithmetic interpreted non-standardly (i.e. as a single cyclic structure without any well-ordering). The latter can be suggested to be 
isomorphic to propositional logic, on the one hand, and to set theory, on the other hand, due to shared structure of Boolean algebra, but eventually under certain conditions to be investigated. Thus, Hilbert arithmetic unlike Peano arithmetic is consistent to set theory, and the Gödel (1931) counterargument is circumvented: Hilbert arithmetic is able to justify mathematics in a consistent way thoroughly within itself therefore guaranteeing completeness.

Thus Hilbert arithmetic embodies the other definitive property of the mathematical totality, that of consistent completeness.

The outlined syllogism transfers the physical and mathematical totality to the other boundary of mathematics, with physics, as a single element (i.e. as a wave function in mathematical terms) of the same totality, already interpreted as the qubit Hilbert space.

The transfer involves as a necessary condition the so-called transcendental invariance due to the separable complex Hilbert space itself, more precisely to its property of unitarity able to identify a class of equivalence what any ordinal number is (in the Cantor - Russell definition) with a certain well-ordering (for example, being granted as "zero" or according to the Neumann definition of ordinal number). In fact, the transfer relies on another fundamental property inherent in mathematics, formulated and well-known in set theory, and hence, available in Hilbert arithmetic (even in as single bit of information as the "fundamental, formal and logical tautology" above demonstrates) as well:

This is the equivalence of the well-ordering "theorem" and the axiom of choice ${ }^{35}$. Then, it allows for the quantity of information (e.g. definable in set theory as the minimal amount of elementary choices necessary for a certain set to be well-ordered, i.e. the cardinal number corresponding equivocally to the ordinal number of the set at issue) to equate a class of equivalence (as what any set can be considered) to any (or each, in a not less degree) element of the same class. In other words, the quantity of information in a set and the same set well-ordered as a series is equal, and even, the same.

That consideration elucidates furthermore the reason for information to be so fundamental in physical and mathematical transcendentalism. On the one hand, it lets to be meant physically what is out of time: for example, what is within the Standard model is within time in the sense of admitting a bijective temporal representation as readings of the apparatus as well. Thus, it does not need information or quantum information, but this is also the reason to "see" quantum information as "dark" field. On the on the hand, it can be interpreted mathematically as an equivalent of the transcendental invariance itself: information means the quantity of doublings (each of which is a bit of information); the doublings of any transcendental element to the pair of a corresponding immanent element and an alternative and disjunctive, corresponding transcendent element.

By doublings and information, the choice - ordering equivalence (i.e. the equivalence of the axiom of choice and the well-ordering "theorem") is observed as the fundamental equality of the number of transcendental elements, the number of immanent elements, and the number of transcendent elements.

Thus, the "root" of dark field is situated still in set theory, but visible only after Hilbert arithmetic in the final analysis. Respectively, one can formulate the concept of dark field even arithmetically, but only after that generalized meaning of "arithmetic". It originates from the identification (though complementary) of Hilbert arithmetic and the separable complex Hilbert space of quantum

\footnotetext{
${ }^{35}$ Both implications are rather elementary and proved in the beginning of the $20^{\text {th }}$ century: the one by Zermelo, the other by Whitehead and Russell.
} 
mechanics. The same link is what connects physics and mathematics by a smooth bridge in their foundations themselves:

The additional amount of information is "hidden" in any qubit, which is both atemporal and aspatial (i.e. omnipresent) by itself, and thus seems to be "dark", if one stretches the temporal cognitive "screen" as physics did until theory of quantum information appear, on that "screen". Speaking more loosely, the conservation of quantum information expresses just that property of its units, the qubits to be omnipresent, i.e. atemporal and aspatial, anyway allowing for a visible "dark" image on the temporal cognitive "screen".

That amount of "dark information" is transformed into the "dark action" of a "dark field" by the mediation of the fundamental Planck constant.

\section{THE SECOND DEDUCTION: FROM QUANTUM INFORMATION CONSERVATION TO DARK ENERGY \& DARK MATTER}

The second deduction inferring both components of dark field from the conservation of quantum information due to the first deduction relies also on the physical and mathematical transcendentalism itself: by identifying physics and mathematics in their foundations, or the transcendental doubling applied to itself. Transcendentalism by itself is doubled as physical transcendentalism and mathematical transcendentalism. This is available yet in the identification of Hilbert arithmetic and the separable complex Hilbert space, after which "dark information" appears by itself necessarily.

That dark information can be understood in terms of philosophy of mathematics as the differential quantity of the information of any certain finite series or set to the information of any also certain infinite series or set. This means that still involving quantum information implies "dark information" in order to be reconciled and then equated mathematically the finite and infinite, or respectively the part and whole physically.

The second deduction is not more than a physical interpretation of the "dark information" proved by the first deduction as the most fundamental law of nature: the conservation of quantum information. The dark information can be justified by that conservation, in virtue of which it appears. And vice versa as well: its experimental confirmation is consistent to it as far as the rejection of dark field (being the physical equivalent of dark information) would refuse quantum-information conservation by the back reflection of modus tollens.

That physical interpretation of dark information as dark field need be understood by mediation of still one postulate referring to what the Planck constant means, namely the physical equivalent of a bit of information into the quantity of action. The Planck constant is too small and the direct experiments to be confirmed or rejected that postulate are too difficult technically for the contemporary experimental science. However, corollaries from them, such as dark field are verified or can be checked.

Furthermore, transcendental invariance verifies the identification of the separable complex Hilbert space of quantum mechanics with the pseudo-Riemannian space of general relativity under a certain condition called "transcendental condition" and embedded in the quantum mechanics itself, or respectively, in the separable complex Hilbert space itself. It represents only a physical interpretation of the choice-ordering equivalence originating still from set theory and inherited in Hilbert arithmetic, which transfers it in the separable complex Hilbert space, from where gets into quantum mechanics. Thus, the condition under which quantum mechanics can be considered as equivalent to general relativity is embedded in the former before that by Hilbert arithmetic, in which the foundations of mathematics and physics are merged. 
Pseudo-Riemannian space is the temporal counterpart of the qubit Hilbert space, the entanglement of the universe (i.e. the physical totality) is transferred and translated in pseudo-Riemannian space and its language (namely Einstein's "Mach's principle") as dark "gravitational field" consisting by space-time component ("dark matter") and energy-momentum component ("dark energy").

The pathway outlined by both deduction is paved and ends ...

\section{$\mathrm{X}$ INSTEAD OF CONCLUSION: THE COMPOSITE DEDUCTION AND ITS PHILOSOPHICAL AND CULTURAL SENCE}

The composite deduction means: the totality implies dark matter and dark energy.

The premise of the totality is a philosophical postulate and even unverifiable as such.

On the contrary, the conclusion of both is either confirmable or refutable even experimentally. To be corroborated and established very well is the case.

This does not verifies the premise, but is consistent to it. The abyss originating from Descartes and predetermining the modern Western philosophy and science has to be overcome by merging the foundations of physics and mathematics, and even philosophy in the totality and reflected by transcendentalism.

\section{References:}

Ade, P. A. R. et al. (Planck Collaboration) (2016) "Planck 2015 results. XIII. Cosmological parameters," Astronomy and Astrophysics 594 A13: 1-63.

Dick, Steven J. (2006) "Anthropology and the search for extraterrestrial intelligence: An historical view," Anthropology Today 22 (2): 3-7.

Dirac, Paul A. M. (1931) "Quantised singularities in the electromagnetic field," Proceedings of the Royal Society of London, A133 (821): 60-72 http://doi.org/10.1098/rspa.1931.0130

Einstein, Albert (1905) "Zur Elektrodynamik bewegter Körper," Annalen der Physik 4 (17) [322 (10)]: 891-921.

Einstein, Albert (1918) “Prinzipielles zur allgemeinen Relativitätstheorie," Annalen der Physik 55 (4) [360 (4)]: 241-244.

Einstein, Albert (1920) Äther und Relativitätstheorie: Rede gehalten am 5. Mai 1920 an der ReichsUniversität zu Leiden, Berlin, Springer.

Einstein, Albert (1926) "Letter to Max Born, 16 December 1926," in Albert Einstein Max Born Briefwechsel 1916 - 1955 (kommentiert von Max Born), München, Nymphenburger Verlagshandlung, 1969: pp. 129-130.

Feingold, Mordechai (2004) "Giordano Bruno in England, Revisited," Huntington Library Quarterly 67 (3): 329-346

Gatti, Hilary (1989). The Renaissance Drama of Knowledge. Volume 3. Giordano Bruno in England. London and New York, Routledge, 2013.

Hart, Michael H. (1975) "An Explanation for the Absence of Extraterrestrials on Earth," Quarterly Journal of the Royal Astronomical Society 16 (6): 128-135.

Howell, Elizabeth (2018 April 17) "Fermi Paradox: Where Are the Aliens?" Space.com, https://www.space.com/25325-fermi-paradox.html (seen October 10, 2020).

Kochen, Simon and Specker, Ernst P. (1967). "The problem of hidden variables in quantum mechanics". Journal of Mathematics and Mechanics 17 (1): 59-87

The Nobel Committee (2011) “The Nobel Prize in Physics 2011”, NobelPrize.org, Nobel Media AB 2020. https://www.nobelprize.org/prizes/physics/2011/summary/ (seen Oct 10, 2020).

Massa, Daniel (1977) "Giordano Bruno's Ideas in Seventeenth-Century England," Journal of the History of Ideas 38 (2): 227-242 
McMullin, Ernan (1986) "Giordano Bruno at Oxford," Isis 77 (1): 85-94.

McNulty, Robert (1960) "Bruno at Oxford," Renaissance News 13 (4): 300-305.

Von Neumann, Johan (1923), "Zur Einführung der transfiniten Zahlen," Acta litterarum ac scientiarum Ragiae Universitatis Hungaricae Francisco-Josephinae, Sectio scientiarum mathematicarum, 1: 199-208

Von Neumann, John (1932) Mathematische Grundlagen der Quantenmechanik, Berlin, J. Springer.

Pellegrini, Angelo M. (1942) "Giordano Bruno and Oxford," Huntington Library Quarterly 5 (3): 303 316.

Penchev, Vasil (2009) Philosophy of Quantum Information. Volume I. Einstein and Gödel. Sofia, IPhR BAS (in Bulgarian, original title "Философия на квантовата информация. Том 1. Айнщайн и Гьодел“). https://philpapers.org/rec/PEN-7

Penchev, Vasil (2010) "The irresolvability of the first incompleteness theorem. Gödel and Hilbert mathematics," Philosophical Alternatives 19 (5): 104-119 (in Bulgarian, original title "Неразрешимост на първата теорема за пълнотата. Гьоделова и Хилбертова математика“"), https://philpapers.org/rec/PEN-21

Penchev, Vasil (2013) "The Kochen - Specker theorem in quantum mechanics: a philosophical comment," Philosophical Alternatives 22 (1): 67-77; 22 (3); 64-73, https://philpapers.org/rec/PENTK-2;

https://philpapers.org/rec/PENTK

Penchev, Vasil (2020 June 29) "All Science as Rigorous Science: The Principle of Constructive Mathematizability of Any Theory," Logic \& Philosophy of Mathematics eJournal (Elsevier, SSRN) 12 (12): 1-15, https://dx.doi.org/10.2139/ssrn.3618926

Penchev, Vasil (2020 July 10) "Quantum Information as the Information of Infinite Series," Information Theory \& Research eJournal (Elsevier, SSRN) 1 (14): 1-8, https://dx.doi.org/10.2139/ssrn.3630063

Penchev, Vasil (2020 July 17) "From the Four-Color Theorem to a Generalizing 'Four-Letter Theorem': A Sketch for 'Human Proof' and the Philosophical Interpretation," Logic \& Philosophy of Mathematics eJournal (Elsevier, SSRN) 12 (21): 1-10, https://dx.doi.org/10.2139/ssrn.3635195

Penchev, Vasil (2020 August 10) "Fermat's Last Theorem Proved by Induction (and Accompanied by a Philosophical Comment)," Metaphilosophy eJournal (Elsevier, SSRN) 12 (8): 1-8, https://dx.doi.org/10.2139/ssrn.3648127

Penchev, Vasil (2020 August 25) "The relationship of arithmetic as two twin Peano arithmetic(s) and set theory: A new glance from the theory of information," Metaphilosophy eJournal (Elsevier, SSRN) 12 (10): 1-33, https://dx.doi.org/10.2139/ssrn.3656179

Penchev, Vasil (2020 October 5) "Quantum-information conservation. The problem about "hidden variables", or the "conservation of energy conservation" in quantum mechanics: A historical lesson for future discoveries," Energy Engineering (Energy) eJournal (Elsevier, SSRN) 3 (78): 1-27.

https://dx.doi.org/10.2139/ssrn.3675319

Riess, A. et al. (Supernova Search Team) (1998) "Observational evidence from supernovae for an accelerating universe and a cosmological constant," Astronomical Journal 116 (3): 1009-1038.

Shostak, Seth (2001 October 25) "Our Galaxy Should Be Teeming With Civilizations, But Where Are They?" Space.com, http://space.com/searchforlife/shostak_paradox_011024.html (seen October 10, 2020).

Trimble, V. (1987) "Existence and nature of dark matter in the universe," Annual Review of Astronomy and Astrophysics 25 (1): 425-472.

Verlinde, Erik (2011) "On the origin of gravity and the laws of Newton," Journal of High Energy Physics 29 (4): 1-24. https://doi.org/10.1007/JHEP04(2011)029

Yates, Frances A. (1939) "Giordano Bruno's Conflict with Oxford," Journal of the Warburg Institute 2 (3): $227-242$. 